Revue d'archéologie préhistorique

\title{
L'ours qui a vu l'homme ? Étude archéozoologique et taphonomique du site paléolithique moyen de Regourdou (Montignac, Dordogne, France)
}

Nadia Cavanhié

\section{OpenEdition}

Journals

Édition électronique

URL : http://journals.openedition.org/paleo/1742

DOI : $10.4000 /$ paleo. 1742

ISSN : 2101-0420

Éditeur

SAMRA

\section{Édition imprimée}

Date de publication : 1 mars 2011

Pagination : 39-63

ISSN : 1145-3370

\section{Référence électronique}

Nadia Cavanhié, «L'ours qui a vu l'homme ? Étude archéozoologique et taphonomique du site paléolithique moyen de Regourdou (Montignac, Dordogne, France) », PALEO [En ligne], 21 | 2009-2010, mis en ligne le 21 octobre 2011, consulté le 07 juillet 2020. URL : http://journals.openedition.org/ paleo/1742 ; DOI : https://doi.org/10.4000/paleo.1742

\section{(c) (i) (3)}

PALEO est mis à disposition selon les termes de la licence Creative Commons Attribution - Pas d'Utilisation Commerciale - Pas de Modification 4.0 International. 


\title{
L'OURS QUI A VU L'HOMME ? Étude archéozoologique et taphonomique du site paléolithique moyen de Regourdou (Montignac, Dordogne, France)
}

\author{
Nadia CAVANHIÉ (1)
}

\begin{abstract}
Résumé : Le squelette d'un Néandertalien, découvert en 1957 (couche IV), fait de Regourdou (Montignac, Dordogne) un site d'un grand intérêt archéologique. Cette importance est renforcée par l'abondance de l'Ours brun (Ursus arctos), taxon plutôt inhabituel dans un gisement paléolithique. Le site de Regourdou reste pourtant méconnu, ayant souffert d'un historique mouvementé et d'un contexte scientifique particulier.

L'étude du matériel faunique, en partie inédit tant du point de vue de la caractérisation des taxons (déterminations, inventaires -NR, NME, NMI- par couche), qu'archéozoologique et taphonomique (structure démographique de la population ursine, représentation squelettique, fragmentation, altérations, traces d'origine animale et humaine), s'avérait nécessaire pour appréhender la fonction de ce site. Cette étude, centrée sur les grands Carnivores, a permis de mettre en évidence une représentation squelettique équilibrée de tous les éléments osseux pour les ours, ainsi qu'un charognage des os longs par les ours eux-mêmes. Néanmoins, l'exploitation de l'Ours par les Moustériens est attestée par la présence de stries de découpe sur trois ossements. La faible fréquence de ces stries laisse penser à une interaction Homme/Ours ponctuelle, majoritairement à l'intérieur de la couche IV sensu lato, qui semble correspondre au modèle connu pour la majorité des sites moustériens avec exploitation de l'Ours (Ursus arctos et surtout spelaeus).
\end{abstract}

Mots-clés : Moustérien, Regourdou, archéozoologie, taphonomie, Ursus arctos, charognage, stries de découpe.

Key-words: Mousterian, Regourdou, zooarchaeology, taphonomy, Ursus arctos, scavenging, cut marks.

\section{Abridged english version}

The bear that saw Man? Zooarchaeological and taphonomic study of the Middle Palaeolithic Regourdou's site (Montignac, Dordogne, France).

The quasi complete skeleton of the Neanderthal (fig. 2) of Regourdou (Montignac, Dordogne) has been discovered in 1957 by the owner of the property on which the cave sits, Roger Constant. He also searched the original entrance of Lascaux, which is located close to Regourdou (only 500m away). After the supposed disappearance of the skull, the excavation of this collapsed cave was entrusted in 1960 by Eugène Bonifay and Bernard Vandermeersch (fig. 1). However, the excavation was stopped in 1965 by the owner; thus, no complete study of the abundant fauna materiel, which is dominated by of brown bears (Ursus arctos), was published by the excavators. Studies have been conducted on the microfauna (Chaline 1972; SimardPélissier 1966), mesofauna (Donard 1982) and Artiodactyls (Delpech 1996) (tabl. 1) at the site for paleoclimate reconstitution. In addition, brown bears from level IV were studied with a taphonomic point of view. These studies relevealed important divergences in the interpretations of the origin of the site. The excavators ascribed an anthropic origin to the presence of bears at the cave. According to them, Mousterian people linked the bear to the belief in resurrection because of his capacity for hiber-

(1) TRACES, UMR 5608, Maison de la Recherche, Université Toulouse le Mirail, 5 allées Antonio Machado, 31058 Toulouse Cedex 9 nadia.cavanhie@univ-tlse2.fr ou nadia.cava@gmail.com 
nation, and consumed it in the cave during funeral meals. The Mousterian people would then deposit the skins, the head and the paws in structures (pits and tumuli). In the opinion of the excavators, the relationship between Mousterian people and bears was visible in the Neanderthal grave because two bear shin bones were placed in the same position as the Neandertal shin bones, which were absents. In addition, this grave (tumulus IVA) was associated with a pit (IVC), which for the excavators, represented an inhumation of a bear after ritual consummation (fig. 3) (Bonifay E. 2002; Bonifay E. et Vandermeersch 1962; Bonifay E. et al. 2007; Bonifay M.F. 1989). For these authors, the bear bone accumulations are wholly anthropic, but for others authors, a natural mortality (during hibernation) can be envisioned (Fosse et al. 2002).

To participate in this debate, it seemed essential to accomplish a zooarchaeological and taphonomic study on all of the faunal material from the site : a definite bone number count (number of rests -NR-, minimal number of individuals -MNI, minimal number of element -MNE-) (tabl. 2) has been done. In addition, an estimation of the Minimun Number of Skeletal Elements (tabl. 6) and Minimum Number of Individuals (tabl. 2), and an elaboration of the demographic structure (age and sex) of the bear population has been completed. The demographic study was possible thanks to the existence of a lot of sexual dimorphism, which is visible in the bear teeth. Also the age of the bears was estimated thanks to actual observations of the sequences of eruption and dental expulsion (Dittrich 1960) on captive animals. For the adults, the age was calculated according to the degree of dental wear (Quiles 2003). Sex-ratio was established by an osteometric study based on two methods: measurement of the thickness in the dentine enamel junction of the canines (Koby 1949), antéro-later diameter of canines and anterior-posterior and buccolingual dimensions of both molar first ones (Stiner 1998).

Since the herbivorous species having only recently been found, this study is focused only on the carnivorous material, notably of brown bears who dominate the material, in number of rests (NR : $65 \%$ ) and in number of individuals (MNI : $40 \%$ ). In spite of problems of reliability in the precise location (tabl. 3) and the realization of numerous readjustments (matching, refiting and reassembling) inside the bony material (fig. 4 and tabl. 4), this study highlights the small but well balenced sample of all of the bony elements for the bears, all the paired elements (long bones, carpals, tarsals) being represented by an approximately the same number of lefts and rights, and in a quasi identical manner inside levels and structures (tabl. 5). The first taphonomic study (Bonifay M.F. 1989) showed a different of bones (thoracic skeleton in levels, bone of the members in structures). In contrast, found a homogeneous skeletal presentation indoors and outside structures.

The study of demographic structure, established from the dental elements, shows an overrepresentation of the males (fig. 6) and a predominance of juveniles (fig. 5), with other age groups not as well represented. The mortality pattern (fig. 5) does not look like that of a living population (minority adults, majority young and old individuals). On the contrary it seems catastrophic. This taphonomic study brought to the forefront an important bear scavenging assemblage (planche I), which was accumulated by the bear himself. This site came be compared to other sites such as Balme in Collomb (fig. 9) where the bear (Ursus spelaeus here) was the only carnivorous species able to modify the material. Scavenging activity is present through out the stratigraphy, with a concentration in level IV (level of the burial) (fig. 10).

This scavenging activity shows a more or less important occupation by bears. These observations, associated to a catastrophic mortality pattern, seem to suggest a natural mortality pattern for the bears in situ (den or trap?). Nevertheless, bears' exploitation by prehistoric men is confirmed by the presence of cut marks on three bones, one shin bone (fig. 6) and two ribs (fig. 8). The low frequency of those cut marks suggests a punctual interaction between Neandertals and bears, predominantly inside layer IV, which seems to correspond to the model known for the majority of the Mousterian sites with bears exploitation (Ursus arctos and especially spelaeus). Indeed, the accumulations of bear bones with an anthropic origin are extremely rare; the sites that provide proof of bear exploitation are mixed sites, where natural mortality and human intervention can coexist, even in a same level, thus, it's difficult to determine if the bones were accumulated by hunting or predation. Only two sites from the Middle Palaeolithic, Biache-Saint-Vaast (Pas de Calais, France) (Auguste 1992, 1995a, 1995b) and Taubach (Deutschland) (Bratlund 1999; Kürten 1972), were interpreted as brown bear hunting sites, in which the bears hunted in mass. The site of Regourdou does have certain similarities to other sites, such as Hayonim (Israël), Moscerini, Sant'Agostino et Polesini (Italie) (Stiner 1994), in which an interaction between Neandertals and bears seems to have only been punctual.

\section{Introduction}

Le gisement de Regourdou (et non du Régourdou, cf. Maureille et al. 2001) a célébré en septembre 2007 le cinquantenaire de la découverte de la sépulture néandertalienne. Ce site préhistorique reste peu connu du grand public, en dépit de l'extraordinaire matériel archéologique, attribué au Moustérien, qu'il a livré : un squelette néandertalien quasi complet, une industrie lithique de type Quina relativement rare mais homogène et un matériel faunique abondant, dominé par les restes d'ours bruns (Ursus arctos). Contrairement à celle de l'Ours des cavernes (Ursus spelaeus), la dominance de ce taxon est plutôt inhabituelle dans un site du Paléolithique.

Les hypothèses sur l'histoire taphonomique de ce matériel osseux divergent fortement selon les auteurs : interprété comme un lieu où l'Ours aurait joué un rôle symbolique 
fort, le Regourdou est encore aujourd'hui signalé par certains comme un lieu de «Culte de l'Ours » (Lajoux 1996), même si l'origine anthropique du matériel est contestée par d'autres.

Le but de cet article est de participer à cette discussion, l'étude du matériel faunique devant permettre d'identifier le ou les agent(s) responsable(s) de ces accumulations osseuses. En effet, le matériel osseux a fait l'objet d'études paléontologiques (Simard-Pelissier 1966 et Chaline 1972 pour la micro et méso faune, Donard 1982 pour le Lièvre en particulier) et biochronologique (Delpech 1996 pour les Artiodactyles). Une seule étude taphonomique, axée sur la structure démographique (sexe et âge), a été menée sur le matériel des Ursidés de la couche IV (Bonifay M.F. 1989, 2008) : il semblait donc nécessaire de reprendre l'étude du matériel osseux, resté en grande partie inédit.

\section{1 - Présentation du gisement et travaux antérieurs}

\section{1 - Bref historique}

En 1954, Roger Constant commence à creuser sans autorisation le terrain qu'il vient d'hériter de son oncle, à la recherche de l'entrée originelle de la grotte ornée de Lascaux, proche d'environ $500 \mathrm{~m}$. Une autorisation de fouilles lui est accordée en 1956 par François Bordes (alors Directeur des Antiquités Préhistoriques d'Aquitaine) grâce à sa découverte d'un gisement moustérien dans une grotte effondrée. Suite à la mise au jour, dans la nuit du 22 au 23 septembre 1957, d'un squelette néandertalien et la disparition présumée de la calotte crânienne, le propriétaire du site reçoit l'interdiction de poursuivre ses fouilles mais entreprend de gigantesques travaux de désobstruction à proximité immédiate du gisement même. Après s'être assuré de la sauvegarde du précieux squelette en envoyant anthropologues et préhistoriens sur place, F. Bordes confie en 1960 l'étude du site à Eugène Bonifay, assisté de Bernard Vandermeersch, alors étudiant en anthropologie. Néanmoins, R. Constant, toujours propriétaire du terrain, continue à s'opposer à ce qu'il considère comme une expropriation, entraînant l'arrêt des fouilles en 1965. Pensant cet arrêt temporaire, les fouilleurs ont reporté la publication du matériel, espérant pouvoir l'appréhender dans sa globalité.

\section{2 - Stratigraphie et structures}

Bien que le site n'ait été fouillé que sur un tiers de sa superficie environ, sa stratigraphie, divisée en deux séquences, a pu être établie (fig. 1) :

- un ensemble supérieur, principalement constitué de blocs rocheux effondrés de l'ancienne voûte de la grotte (couche I) et de sables épais de un à trois mètres (couche II) ; - un ensemble inférieur (d'une puissance stratigraphique de 2,30 à 2,70 mètres) qui s'étend des couches III à VIII et s'arrête à la couche $R$, substrat rocheux créé par d'énormes dalles effondrées de la voûte. L'épaisseur de la couche IV, couche de la sépulture, varie de 30 à $50 \mathrm{~cm}$.

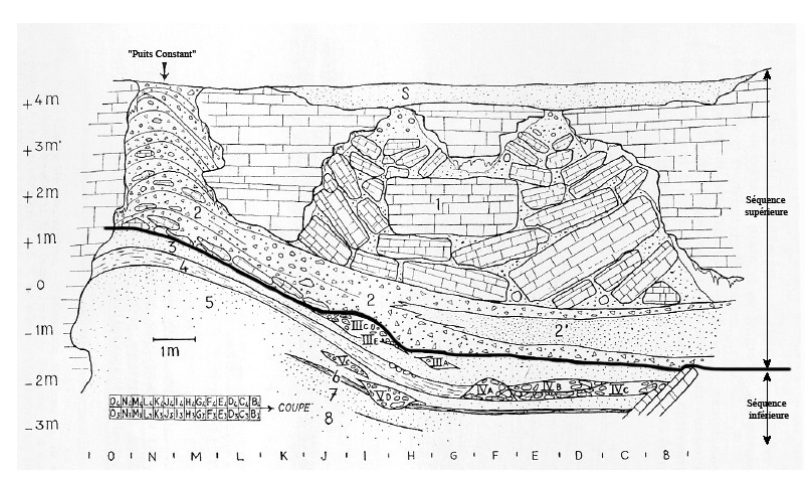

Figure 1 - Stratigraphie partielle de Regourdou (d'après E. Bonifay 2002 - p. 249, fig.2, modifié).

Figure 1 - Regourdou partial stratigraphy (Bonifay E. 2002 p. 249, fig. 2, modified).

La nature principalement sableuse du sédiment a permis l'excellente conservation de la totalité du matériel, les sables varvés ayant emballé les ossements (Bonifay $\mathrm{E}$. 2002 - p. 250).

La séquence inférieure a livré un important matériel archéologique ainsi que de nombreuses structures (sept fosses, quatre pierriers et deux tumuli), dont l'origine anthropique et l'intentionnalité seraient attestées par les observations des fouilleurs (Bonifay et Vandsermeersch 1962 - p. 1636) : disposition du matériel, structures de protection (dallages de pierre, coffrage interne protégeant un crâne d'ours...), pierres rapportées de l'extérieur... Les deux tumulus (VIIA et IVA) montrent une construction similaire : hauts de près d'un mètre, ils sont tous deux constitués par un tas de pierres, de sables et de cendres, le tumulus IVA (sépulture humaine) étant couronné par les restes d'un petit foyer. Sur les treize structures recensées, neuf ont livré du matériel archéologique ou paléontologique, principalement constitué de restes osseux (crânes et os longs majoritairement) d'ours bruns.

\section{3 - Attribution chronologique du gisement}

Les datations réalisées à l'université de Groningen (Allemagne) ayant échoué, donnant une date de 8000 ans BP pour le squelette néandertalien, seule une attribution chrono-culturelle a pu être établie à partir de plusieurs études (géologie, paléoanthropologie, matériel lithique, faunes, etc.). En se basant sur l'étude géologique et sédimentologique du gisement (remplissages, phénomènes cryoclastiques, etc.), E. Bonifay a proposé une datation relative des couches (Bonifay E. et al. 2007 - p. 6). Selon lui, les structures des couches IV à VIII auraient été réalisées durant le Würm I (90 à 60000 BP). La couche III serait contemporaine de l'interstade Würm I/Würm II (60 à 55000 ans BP), et la couche II du Würm II, qui marquerait la fin de l'histoire archéologique de Regourdou avec un nouvel effondrement du plafond, puis son affaissement 
complet, rendant la grotte inhabitable à la fin de cet épisode glaciaire. Cette attribution du gisement au Würm ancien est confirmée par l'interprétation des associations de micro et méso mammifères (Chaline 1972 - p. 230-231), ainsi que par l'étude des Artiodactyles menée par F. Delpech, qui propose de corréler la couche II du gisement au stade isotopique 4, en raison, notamment, de la présence d'une faune froide typique, et la couche IV au stade isotopique 5 (Delpech 1996). L'industrie lithique, décrite comme appartenant à un Moustérien de type Quina, ne va pas à l'encontre de ces interprétations (Bonifay E. 1963, 2002).

\section{4 - Squelette humain}

Le matériel mis au jour est d'une grande importance archéologique, notamment le squelette découvert dans le tumulus IVA, qui fait de Regourdou, au moment de sa découverte, un site majeur dans la connaissance de Néanderthal, cette inhumation étant parfois considérée comme la plus ancienne sépulture d'Europe (Maureille et Vandermeersch 2007 ; Turq et al. 2008).

De nombreuses études détaillées ont porté sur ce squelette (fig. 2), notamment sur ses caractéristiques anatomiques et son degré d'évolution (voir références in Madelaine et al. 2008). Elles ont permis de déterminer que ces restes humains appartiennent majoritairement à un Néandertalien évolué, de sexe indéterminé, dont l'âge est estimé à moins de 25 ans (Regourdou 1). Certaines observations (réalisation d'un tumulus d'un mètre de haut (IVA), peut-être associé à la fosse IVC contenant les restes d'une ourse) sembleraient indiquer une grande complexité dans le dépôt du

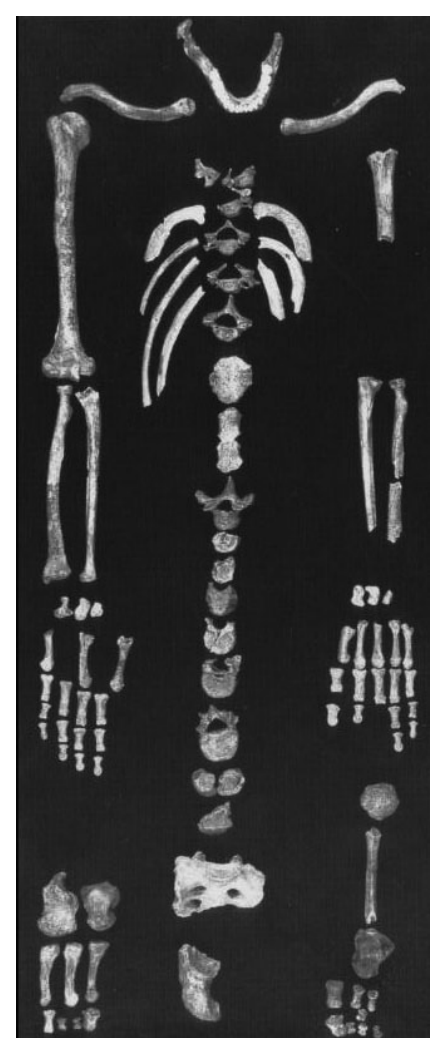

Figure 2 - Squelette néandertalien « Regourdou 1 » (position reconstituée, cliché Bernard Dupuy, collection Musée d'Art et d'Archéologie du Périgord, ville de Périgueux).

Figure 2 - Neandertal skeleton « Regourdou 1 » (reconstructed position, Bernard Dupuy's photography, collection of Museum of Art and Archaeology of Périgord, city of Périgueux). squelette. De plus, ce squelette (Regourdou 1) a été retrouvé quasiment complet. L'absence remarquée du crâne et des deux tibias a donc posé problème, plusieurs explications pouvant être avancées : vol du crâne, phénomènes taphonomiques post-dépositionnels ou remaniements postérieurs à l'inhumation, comparables à ceux observés dans la grotte de Kébara (Israël), dans laquelle les Néandertaliens sont venus rechercher le crâne et les tibias d'un individu inhumé précédemment (Tillier et al. 1991). Un troisième calcanéum, retrouvé hors contexte sépulcral, suggère la présence d'un deuxième individu (Regourdou 2) (Vandermeersch et Trinkaus 1995).

\section{5 - Industrie lithique}

L'industrie lithique, bien que relativement rare, est décrite comme homogène (Bonifay E. 1963). Présente à tous les niveaux, elle est définie comme caractéristique du Moustérien de type Quina, malgré la présence d'un débitage type Levallois : “ il s'agit partout d'un Moustérien charentien de type "La Quina " avec quelques très beaux racloirs à retouche scalariforme et les autres outils caractéristiques de cette civilisation du Paléolithique moyen " (Bonifay E. 2002 - p. 249). Ce matériel lithique est actuellement réétudié par Alain Turq (Musée national de Préhistoire -MNP-, Les Eyzies-de-Tayac, Dordogne).

\section{6 - Restes fauniques}

\subsection{1 - Micro et méso mammifères}

Treize espèces de rongeurs, 3 de lagomorphes, 4 d'insectivores et 2 de chiroptères ont été étudiées (SimardPelissier 1966). L'interprétation des accumulations osseuses de micromammifères comme issues de pelotes de rejection des rapaces (ibid p.4, 71) est contestée (E. Bonifay, com. pers.).

\subsection{2 - Herbivores (tabl. 1)}

Les Artiodactyles, seul ordre étudié (Delpech 1996), sont représentés par 709 restes, principalement concentrés à l'intérieur de la couche II, qui a livré $59 \%$ de ce matériel. Sept espèces, appartenant à trois familles (Suidés, Cervidés et Bovidés), ont été identifiées, parmi lesquelles le Renne domine largement (44\%).

\subsection{3 - Carnivores}

Bien qu'ils soient décrits comme dominant largement le matériel faunique (Bonifay M.F. 1989 - p. 45), aucun décompte précis n'est donné pour les Carnivores, hormis pour les 918 restes osseux d'ours bruns recensés dans la couche IV. Ces derniers ont été attribués à une sousespèce, Ursus arctos aquitanicus, " caractérisée par l'association de dents de taille moyenne à petite, à caractères archaïques, avec un squelette de taille normale " (Bonifay M.F. ibid. et 2008). D'autres espèces sont mentionnées dans le spectre faunique : le Loup et le Lion («Felis spelaea Gold. », Bonifay E. 2002 - p. 248), ainsi que l'Hyène (Fosse et al. 2002 - p. 96). 


\begin{tabular}{|c|c|c|c|c|c|c|c|c|c|}
\hline & I & II & III & IV & V & VI & VII & VIII & Total \\
\hline Sus scrofa & & 64 & 9 & 20 & 3 & & & & $\mathbf{9 6}$ \\
\hline Cervus elaphus & 2 & 59 & & 1 & 1 & & & & $\mathbf{6 3}$ \\
\hline Cervus simplicidens & & 3 & 31 & 61 & 36 & 5 & 14 & & $\mathbf{1 5 0}$ \\
\hline Rangifer tarandus & 1 & 269 & 13 & 25 & 1 & & & & $\mathbf{3 0 9}$ \\
\hline $\begin{array}{c}\text { Capreolus } \\
\text { capreolus }\end{array}$ & & 16 & 8 & 8 & 11 & 1 & 17 & 1 & $\mathbf{6 2}$ \\
\hline Cervidae indét. & & 4 & 1 & 1 & 1 & & & & $\mathbf{7}$ \\
\hline Bos primigenius & & 1 & & 6 & 2 & & 3 & & $\mathbf{1 2}$ \\
\hline Bison priscus & & 2 & & & & & & & $\mathbf{2}$ \\
\hline Bovinae indét. & & 2 & 2 & & 2 & 1 & 1 & & $\mathbf{8}$ \\
\hline TOTAL & $\mathbf{3}$ & $\mathbf{4 2 0}$ & $\mathbf{6 4}$ & $\mathbf{1 2 1}$ & $\mathbf{5 7}$ & $\mathbf{7}$ & $\mathbf{3 5}$ & $\mathbf{1}$ & $\mathbf{7 0 9}$ \\
\hline
\end{tabular}

Tableau 1 - Répartition des restes d'Artiodactyles de Regourdou, par taxon et par couche (d'après Delpech 1996 p. 33, tabl. 1).

Tableau 1 - Complete distribution of artiodactyls remains of Regourdou cave, by taxon and by level (Delpech 1996 - p. 33, tabl. 1).

\section{7 - Origine de ce matériel}

\subsection{1 - Une origine anthropique}

Selon E. Bonifay, la relation Homme/Ours serait la caractéristique principale du site de Regourdou. Cette relation serait particulièrement visible dans la couche IV, notamment à l'intérieur de la sépulture humaine (tumulus IVA), où elle serait signifiée par le dépôt de deux tibias d'ours, qui " prolongeaient le corps vers le bas, à l'emplacement qu'auraient dû avoir [l]es jambes [du Néandertalien] si elles avaient été en extension ", mais aussi par le dépôt d'objets sur un bloc rocheux posé sur la poitrine du mort, dont une partie distale d'humérus d'ours (Bonifay E. 2002 p. 250). Enfin, elle serait confortée par la création d'une superstructure (fig. 3), "véritable monument funéraire 》 (Bonifay E. et al. 2007 - p. 6), associant, par la construction de murs et la pose d'une lourde pierre, la sépulture humaine (tumulus IVA) et la fosse IVC contenant le squelette d'un ours femelle de petite taille (Bonifay M.F. 1989 p. 46). Le squelette est décrit comme « presque complet mais désarticulé avant le dépôt : les os longs étaient rangés le long des grands côtés du rectangle, les deux omoplates étaient (croisées) à l'extrémité sud, tandis qu'au nord le crâne reposait entre trois pierres formant un petit coffrage protecteur »(Bonifay E. 2002 - p. 250).
De plus, l'étude taphonomique réalisée par M.F. Bonifay a mis en évidence une distribution squelettique différentielle (" les restes de squelette thoracique se trouvent principalement hors structure, les os des membres étant dans les pierriers "), traduisant selon elle le dépeçage in situ des ours par les Néandertaliens et le dépôt des " peaux munies de la tête et des pattes osseuses » dans les structures (Bonifay M.F. 1989 - p. 46). L'aspect pratique de ce procédé, attesté par des exemples ethnographiques, a été confirmé expérimentalement (Poplin 1980).

Selon E. Bonifay, le squelette humain de Regourdou pourrait avoir été déposé sur une telle fourrure (Bonifay E. 2002 - p. 250). D'après lui, les marques de découpe et de décarnisation présentes sur les os de la petite femelle de la fosse IVC impliqueraient une "consommation de l'ours au cours d'un repas funéraire (...), au cours de cérémonies liées à des rites funéraires 》 (Bonifay E. 2002 - p. 252).

Néanmoins, les rituels funéraires ne seraient pas limités à la seule couche IV : ainsi, les structures présentes sur le site ont été analysées comme " des dépôts d'ossements d'ours brun qui peuvent être antérieurs (couches V, VI, VII), contemporains (couche IV), ou postérieurs (couche III) à l'inhumation humaine " (Bonifay E. 2002 - p.248). Cette interprétation est basée sur le fait que toutes les structures, hormis quatre fosses n'ayant livré que des cailloux (VD, IVC, IIIC et IIIE), contenaient des restes d'ours isolés (pierrier IVD ; fosse IIIA : quelques bourgeons dentaires d'ourson) ou associés à un ou plusieurs crânes d'ours. De plus, trois structures (tumulus VIIA, fosse VA et fosse IVC) ont livré des éléments de protection des crânes, des coffrets en pierres, grossiers ou au contraire très nets. Selon $E$. Bonifay, les hommes de Néandertal auraient réalisé ces dépôts d'os d'ours pour célébrer la mort d'un des leurs. L'auteur prête en effet au plantigrade une symbolique forte, celle de la résurrection (attribuée également aux bois de cerf présents dans les deux tumuli) : « l'Ours était un symbole puissant qui avait des relations très étroites avec la mort, et l'ours a peut-être évoqué cette autre vie par le fait qu'il hiberne en hiver et réapparaît au printemps, en même temps que s'opère le renouveau de tout le milieu naturel, comme s'il " ressuscitait » après sa disparition momentanée » (Bonifay E. 2008 - p. 29). Ainsi, selon lui, les Néandertaliens auraient déposé, dans la sépulture, os

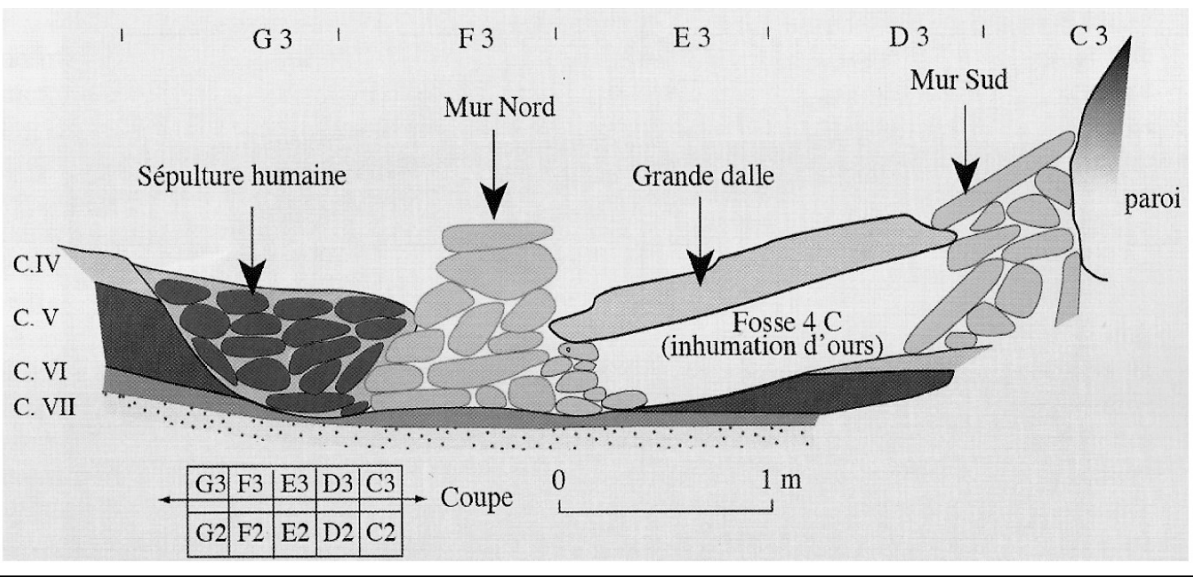

Figure 3 - Coupe longitudinale de la sépulture IVA et de la fosse à ours IVC (Bonifay E. et al. 2007 - p.7, fig.7, modifié).

Figure 3 - Longitudinal section of the grave IVA and the pit IVC (Bonifay E. et al. 2007 - p.7, fig.7, modified). 
d'ours et bois de cerf afin d'assurer la résurrection du seul membre de la tribu inhumé à l'intérieur de la grotte, mais tous les dépôts d'ossements d'ours seraient autant de rites funéraires : " dans la plupart des cas l'homme était enterré hors de la grotte, seule une offrande symbolique (le crâne de l'ours seul, ou le crâne et quelques ossements) étant déposée dans la grotte »(Bonifay E. 2002 - p. 252).

\subsection{2 - Une origine naturelle}

A cette hypothèse d'une origine entièrement anthropique fortement liée au symbolique, d'autres chercheurs, ayant eu accès (au moins partiellement) au matériel osseux, ont opposé l'hypothèse d'une mortalité naturelle, in situ, des Ursidés (Fosse et al. 2002). Ils se basent pour cela sur plusieurs critères, comme les traces de charognage attribuées aux ours eux-mêmes ou la structure démographique de la population, montrant une prédominance des jeunes adultes et sub-adultes, "distribution [qui] pourrait soit être mise en parallèle avec les tanières d'ours bruns actuels (occupation ourse-oursons du deuxième hiverl adultes mâles solitaires), soit évoquer l'éventualité d'un piégeage naturel occasionnel » (Fosse et al. 2002 - p. 94) : “ $A u$ Régourdou, le faible nombre d'individus et la présence de tous les éléments squelettiques ne plaident guère en faveur d'une accumulation d'origine véritablement anthropique. Si les carcasses d'ours avaient été amenées (complètes) et déposées dans un quelconque dessein, il n'y aurait aucune trace d'origine animale (grignotements) sur les os longs. Une autre hypothèse serait de dire que d'autres grands prédateurs (hyène et lion), présents de façon marginale sur le site ont été attirés par les carcasses d'ours, déposées par les Moustériens » (ibid p. 96).

\section{2 - Les grands mammifères : taphonomie et archéozoologie}

\section{1 - Matériel et méthodes : généralités}

\subsection{1- Méthode}

Afin de participer à cette discussion sur l'origine taphonomique du matériel faunique de Regourdou, notamment celui des Ursidés, une étude archéozoologique et taphonomique classique a été menée, commençant donc par l'établissement de décomptes précis (nombre de restes NR-, d'éléments squelettiques -NME- et d'individus potentiellement présents -NMI-) (tabl. 2 et 6 ). Pour des raisons d'accessibilité du matériel, cette recherche s'est centrée sur les restes de grands Carnivores, notamment les Ursidés, conservés dans leur grande majorité au MNP. Plusieurs axes ont été étudiés :

\section{- Représentation squelettique}

Afin de permettre les comparaisons, les portions squelettiques définies pour Ursus arctos lors d'une première étude taphonomique (Bonifay M.F. 1989) ont été gardées : le squelette " crânien 》 est donc composé des crânes, mandibules et dents isolées et le « thoracique » des ver- tèbres, côtes et sternum. La « patte avant » englobe scapula, humérus, radius-ulna, carpiens et métacarpiens, tandis que bassin, fémur, tibia, tarsiens et métatarsiens constituent la « patte arrière ». Enfin, une dernière catégorie, celle des "sésamoïdes ", regroupe les os communs aux membres antérieurs et postérieurs, soit les sésamoïdes et phalanges, auxquels ont été ajoutés les os du métapode (métacarpiens ou métatarsiens impossibles à déterminer).

\section{- Structure démographique de la population ursine}

La structure démographique (en terme de classes d'âge) des Ursidés a été évaluée d'après le matériel dentaire : l'âge des juvéniles a pu être estimé grâce aux observations des séquences d'éruption et d'expulsion dentaire d'animaux captifs (Dittrich 1960), tandis que celui des adultes a été calculé en fonction du degré d'usure dentaire (Quiles 2003 - p.144-148). L'étude des différents éléments dentaires a ainsi permis la constitution de classes d'âge définies classiquement, en fonction des rares informations publiées sur l'éruption dentaire chez l'Ours brun (Dittrich 1960 ; Couturier 1954 ; Donat-Ayache 2003), en " juvéniles 》 (individus âgés de moins d'un an), “ jeunes adultes » (1 à 5 ans), « adultes » (5 à 10 ans) et « vieux adultes » (plus de 10 ans).

Enfin, le fort dimorphisme sexuel existant chez les ours a permis d'établir le sex-ratio grâce à une étude ostéométrique basée sur deux méthodes : mesure du diamètre transverse au collet des canines (Koby 1949), diamètre antéro-postérieur des canines et diamètre antéro-postérieur et bucco-lingual des deux premières molaires (Stiner 1998). Une autre méthode, mise au point par F. Prat et C. Thibault (Prat et Thibault 1976 - p.57), n'a pas été utilisée : en effet, elle consiste à mesurer le périmètre des canines au niveau du collet (à l'aide d'un mince ruban adhésif) et nécessite donc des canines au collet bien marqué, ce qui n'est pas le cas sur le matériel de Regourdou. Enfin, des tentatives d'ostéométrie sur le post-crânien (éléments du carpe et du tarse très fortement liés à la masse, comme les pisiformes et scapho-lunaires - Chagneau 1985 ; Stiner 1998) n'ont donné aucun résultat clair, probablement à cause de la taille réduite de l'échantillon ( 9 pisiformes, 19 scapho-lunaires).

\subsection{2 - Matériel}

L'assemblage faunique du site de Regourdou est constitué de 4331 restes osseux (NRT), dont plus de $91 \%$ ont pu être déterminés anatomiquement et taxonomiquement (NRD) : malgré ce fort taux de détermination, résultant d'une bonne conservation du matériel osseux et d'une relative faible fragmentation d'origine, l'étude a souffert de plusieurs biais. L'étude de la répartition spatiale des vestiges osseux a ainsi été gênée par plusieurs problèmes. Premièrement, la localisation stratigraphique d'un nombre relativement élevé de vestiges (34\% du NRT et près de $30 \%$ du NRD) n'est pas renseignée. Se posent alors les questions d'une part de l'origine exacte de ces restes (fouilles Bonifay ou Constant ?) et, d'autre part, de leur mode de prélèvement (en amas ou en pièces isolées ?). 


\begin{tabular}{|c|c|c|c|c|c|c|c|c|c|c|c|c|c|c|c|c|c|c|}
\hline$\sum_{\bar{Z}}^{\bar{Z}}$ & $\infty$ & $\infty$ & & in $x$ & 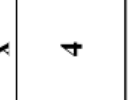 & • & & $\vec{f}$ & & $N$ & & $\nabla$ & & $\Rightarrow$ in & 2 & $*$ & $2=$ & \\
\hline & 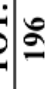 & 6 & & No & $\vec{n}$ & ले & & 氕 & 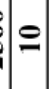 & $\%$ & & $\mathrm{~m}$ & & & ले & & 83 & \\
\hline 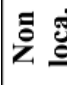 & & ฮे & & \pm & $\infty$ & ते & & స्रे & $\sigma$ & $\cong$ & & $\infty$ & & $\stackrel{\infty}{\circ}$ & 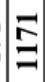 & & $\begin{array}{ll}\vec{y} \\
\vec{y}\end{array}$ & \\
\hline 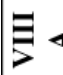 & & $N$ & & - & & $N$ & & $n N$ & & & & & & & $r$ & & r & \\
\hline 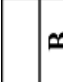 & & - & & - & & - & & $+r$ & & & & & & $r$ & $=$ & & $=\cong$ & 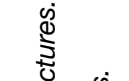 \\
\hline$\geqslant<$ & & $\infty$ & & $N-$ & $m$ & $N$ & & $\Rightarrow f$ & & & & & & F & t & & $\begin{array}{lll}6 & \infty \\
i\end{array}$ & 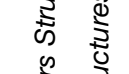 \\
\hline 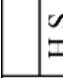 & & in & & $\stackrel{0}{-1}$ & $N$ & - & & $\bar{m} \bar{N}$ & & & & $N$ & & & in & & $60^{\infty}$ & \\
\hline 5 & $N$ & $\nabla$ & & - & - & 4 & & $\theta m$ & & & - & - & & m & $F$ & + & in & \\
\hline$=$ & & $N$ & & & & & & $N-$ & & & & & & & $m$ & - & 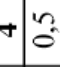 & है \\
\hline 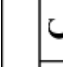 & $m$ & $m$ & & $N$ & & - & & 2 ले & & $\mathrm{N}$ & $1-$ & & & & in & & 馆 & $\frac{0}{0}$ \\
\hline$>\approx$ & $=$ & & & & 4 & & $m$ & $m$ & & - & & & & & $\infty$ & - & $\hat{\imath}$ & 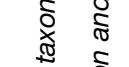 \\
\hline$<$ & 4 & $\nabla$ & & & & & & 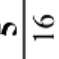 & & & & & & & $\bar{N}$ & & $\bar{N} \sim$ & ฐ \\
\hline$=$ & in & ले & & (6) & in & $\approx$ & & $6: 2$ & - & & - & & & & 곤 & 우 & is & \\
\hline$=$ & - & $m$ & & $-\alpha$ & -1 & - & & $\theta \Re$ & & $m$. & - & & & & $\infty$ & $n$ in & $2 \stackrel{\infty}{\sim}$ & 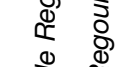 \\
\hline 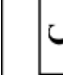 & 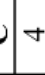 & $\infty$ & & - & - & & & mิ & & - & & & & & ลั & & : & 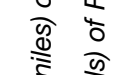 \\
\hline$\geq$ & $=$ in & + & & - & $N$ & & & 9 ) & & - & & & & & సั. & $\sigma$ & $\begin{array}{c}2 \\
\therefore\end{array}$ & \\
\hline$<$ & $4 \infty$ & $\sigma$ & & - & 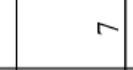 & $a$ & & $\vec{m} \tilde{\sim}$ & & $\sim$ & & 0 & & & लై & $\circ$ & $m$ & 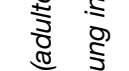 \\
\hline 5 & if & $\exists$ & & $\therefore-$ & $\mathrm{N}$ &.+ & & $\stackrel{\circ}{\stackrel{2}{N}}$ & & $\sim$ & & - & & & 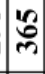 & & ले & 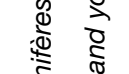 \\
\hline$\approx$ & & & & & & & & 00 & & - & & & & & - & & - & $\frac{0}{3}$ \\
\hline$\equiv<$ & & $m$ & & - & & - & & $0 \simeq$ & & . & - & & & & สิ & & $\vec{\wedge}$ & \\
\hline & $\because$ & $\approx$ & & $r$ & $\nabla$ & in & & $N \cong$ & & $=$ & & + & - & & 今ิ & & 空 & \\
\hline$=$ & $\Rightarrow$ & $\stackrel{\infty}{\curvearrowright}$ & & $=n$ & - & $\overline{0}$ & & ثิ઼ & & 0 & ज. & $\because$ & & & $\sqrt{6}$ & $=\$$ & $: 3$ & 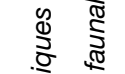 \\
\hline- & & $\mathrm{N}$ & & & & $\mathrm{N}$ & & $+N$ & & & - & & & & $r$ & & $r$ & \\
\hline & & 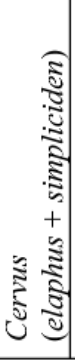 & 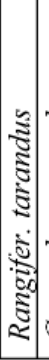 & 's. & 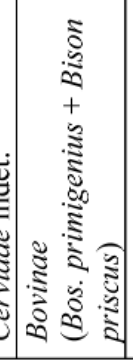 & 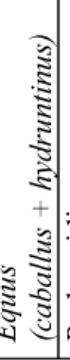 & 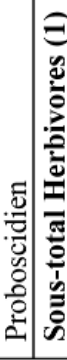 & 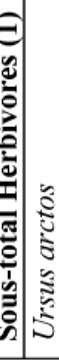 & & |. & & 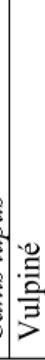 & 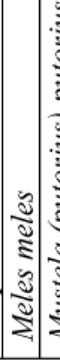 & 先 & 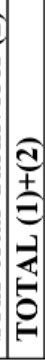 & : & : & 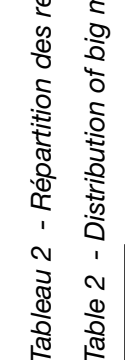 \\
\hline
\end{tabular}


De plus, d'importants problèmes de corrélation entre la base de données initiale, élaborée par E. et M.F. Bonifay, et celle mise au point pour cette étude, sont apparus pour les restes localisés (tabl. 3), la provenance du matériel n'étant certaine que pour un peu plus d'un tiers du matériel localisé. Toutefois, la catégorie " probable » (cotation identique mais détermination anatomique divergente selon le listing) est très certainement le reflet du mode de prélèvement des vestiges (en amas). Reste le problème des 724 restes dont la provenance est « douteuse » (cotation différente selon les listings) : pour savoir si le problème de cote est dû à une erreur lors du marquage des objets ou lors de la saisie dans la base de données initiale, une étude de l'analyse spatiale avec le logiciel Data Desk a été initiée.

Enfin, de nombreux appariements, raccords et remontages ont été réalisés (fig. 4), majoritairement pour les Ursidés (la dispersion géographique du matériel des Herbivores entre plusieurs sites ayant gêné l'étude de leur répartition spatiale) : ces réajustements (tabl. 4) laissent penser que la stratigraphie a subi certaines perturbations qui devront être

\begin{tabular}{|c|c|c|c|}
\hline & SURE & PROB. & DOUT. \\
\hline I & 0 & 0 & 7 \\
\hline II & 81 & 428 & 148 \\
\hline III & 33 & 98 & 76 \\
\hline IIIA & 3 & 12 & 5 \\
\hline IIIB & 0 & 0 & 1 \\
\hline IV & 86 & 147 & 133 \\
\hline IVA & 141 & 83 & 37 \\
\hline IVB & 169 & 50 & 47 \\
\hline IVC & 215 & 46 & 36 \\
\hline IVD & 40 & 24 & 23 \\
\hline V & 51 & 126 & 94 \\
\hline VA & 1 & 16 & 5 \\
\hline VB & 3 & 19 & 6 \\
\hline VC & 13 & 24 & 17 \\
\hline VD & 0 & 3 & 0 \\
\hline VI & 14 & 16 & 17 \\
\hline VII & 3 & 17 & 34 \\
\hline VIIA & 24 & 13 & 27 \\
\hline VIIB & 1 & 6 & 4 \\
\hline VIIIA & 0 & 0 & 7 \\
\hline NRT & $\mathbf{8 7 8}$ & $\mathbf{1 1 2 8}$ & $\mathbf{7 2 4}$ \\
\hline \% & $\mathbf{3 2 , 2}$ & $\mathbf{4 1 , 3}$ & $\mathbf{2 6 , 5}$ \\
\hline
\end{tabular}
Regourdou. Regourdou. étudiées et interprétées. Malheureusement, la rareté des données stratigraphiques $(X, Y, Z)$ sur ces réajustements rend toute interprétation synthétique délicate, notamment en ce qui concerne les réajustements inter couches (matériel réellement éloigné ou interface de 2 couches ?). Toutefois, il est intéressant de constater le nombre important d'appariements, remontages et raccords réalisés à l'intérieur de la couche IV, mais entre des vestiges situés dans et hors structures (ex : couche IV hors structure et tumulus IVA) : cette observation pourrait signifier une certaine porosité de ces structures, une ouverture permettant la circulation des vestiges.

\section{2 - Détermination taxonomique}

Ces données ne concernent que la grande faune et excluent donc les 22 espèces de micro et méso mammifères identifiées dans le site (Simard-Pelissier 1966), ainsi que le Castor (2 hémi mandibules à l'intérieur du pierrier IVD et 4 dents isolées non localisées) et les lagomorphes, représentés par de très nombreux ossements restés en partie inédits (Donard 1982). Bien que cet article ne porte pas sur la paléoanthropologie, il est intéressant de noter que le réexamen du matériel de Regourdou, associé au récolement effectué au MNP, a permis la reconnaissance d'une dizaine de restes humains inédits (Madelaine et al. 2008), complétant de façon significative les membres inférieurs du squelette « Regourdou 1 ».

L'étude du matériel faunique de Regourdou a mis en évidence un spectre faunique très large, puisque 17 espèces animales sont représentées : 9 d'Herbivores et 8 de Carnivores (tabl. 2).

Avec 1201 restes, les Herbivores constituent un tiers $(30,3 \%)$ des ossements déterminés (NRD). Une précédente étude avait permis de mettre en évidence la présence de 6 espèces d'Artiodactyles (Delpech 1996),

Tableau 3 - Degré de fiabilité sur la localisation exacte des vestiges osseux de

Table 3 - Degree of reliability on the precise location of the faunal remains of

\begin{tabular}{|c|c|c|c|c|c|c|}
\hline & $\begin{array}{c}\text { Intra } \\
\text { couche }\end{array}$ & $\begin{array}{c}\text { Couche }+ \\
\text { structures } \\
\text { (même niveau) }\end{array}$ & $\begin{array}{c}\text { Couches } \\
\text { adjacentes }\end{array}$ & $\begin{array}{c}\text { Inter } \\
\text { couches }\end{array}$ & $\begin{array}{c}\text { 1 vestige } \\
\text { minimum non } \\
\text { localisé }\end{array}$ & TOTAL \\
\hline Appariements & 35 & 17 & 11 & 8 & 18 & $\mathbf{8 9}$ \\
\hline Remontages & 18 & 13 & 12 & 6 & 14 & $\mathbf{6 3}$ \\
\hline Raccords & 14 & 4 & 2 & 0 & 21 & $\mathbf{4 1}$ \\
\hline TOTAL & $\mathbf{6 7}$ & $\mathbf{3 4}$ & $\mathbf{2 5}$ & $\mathbf{1 4}$ & $\mathbf{5 3}$ & $\mathbf{1 9 3}$ \\
\hline
\end{tabular}

Tableau 4 - Récapitulatif des réajustements réalisés sur le matériel faunique de Regourdou.

Table 4 - Summary of readjustments accomplished on the fauna material of Regourdou. 

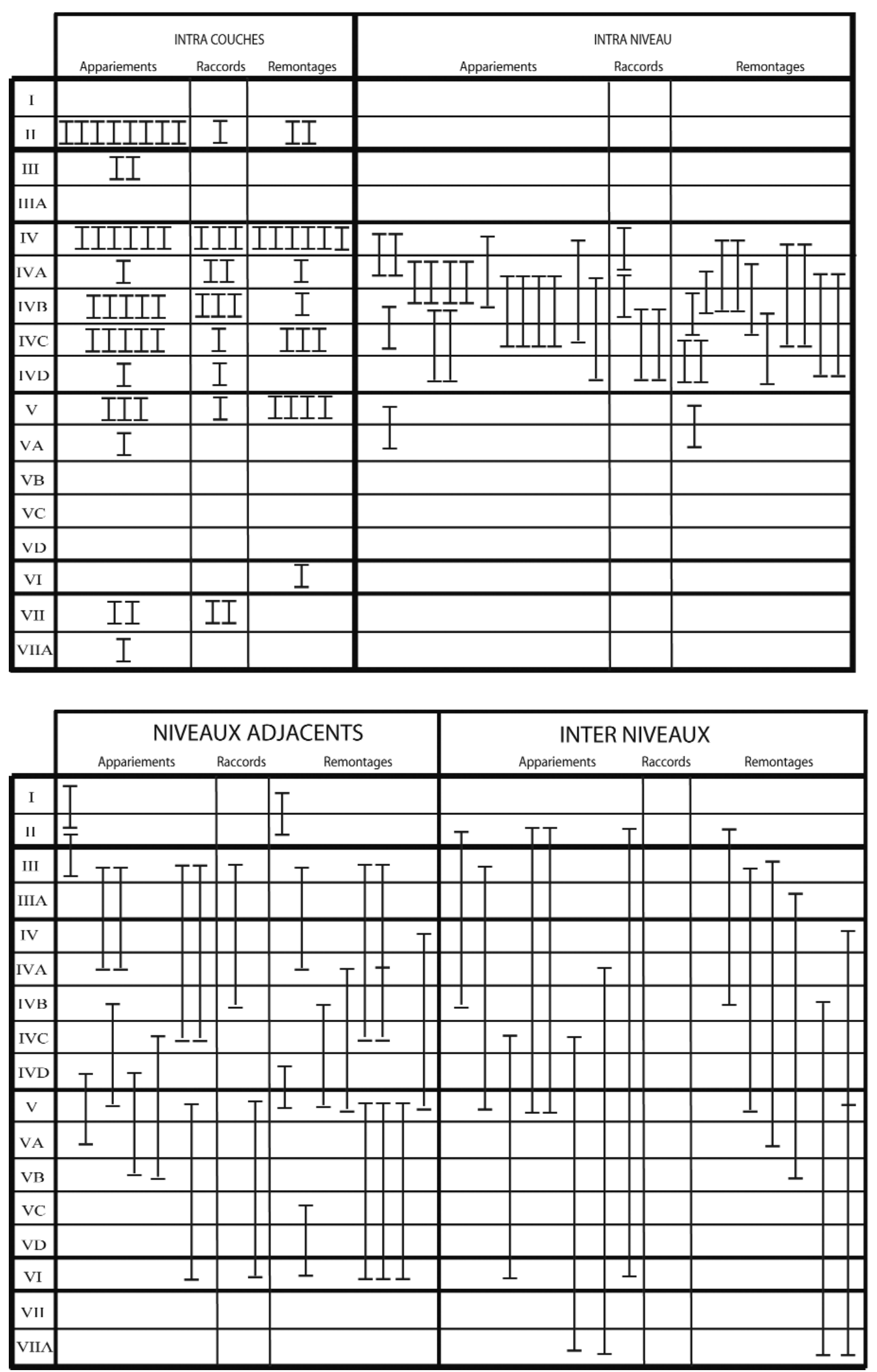

Figure 4 - Provenance spatiale des appariements, raccords et remontages réalisés sur le matériel faunique de Regourdou. Figure 4 - Origin of matching, refiting and reassembling accomplished on the fauna material of Regourdou. 
auxquelles s'ajoutent désormais un ou deux Périssodactyles du genre Equus (coexistence probable de $E$. caballus et hydruntinus) et un Proboscidien, représenté par une phalange qui, de par sa taille, appartiendrait plus probablement à Elephas antiquus qu'à Mammuthus trogontherii (M.F. Bonifay, d'après M. Beden, com. pers.). Ce matériel faunique est nettement dominé (presque $68 \%$ ) par les restes de Cervidés, dont plus de $30 \%$ de Renne. Viennent ensuite le Sanglier (16,3\%), suivi du Cheval, qui représente, avec 139 restes osseux, près de $12 \%$ de ce matériel. vingt restes osseux pourraient être attribués à un Equus immature mais ont été par prudence, en l'absence de collection de comparaison, classés parmi les « indéterminés ». Enfin, les Bovinés (Bison et Aurochs associés) représentent environ $4 \%$ du matériel total des Herbivores.

Les Carnivores sont représentés par 2763 restes (près de $70 \%$ du NRD) appartenant à 8 espèces. Ursus arctos est bien évidemment au premier plan avec 2560 restes, représentant plus des deux tiers du matériel faunique $(64,6 \%$ du NRD) et plus de $92 \%$ du matériel des Carnivores. La présence d'Ursus spelaeus serait éventuellement attestée par une ulna retrouvée en couche $V$ et par quelques ossements présentés dans les vitrines du Musée de Regourdou, sans qu'il soit possible de savoir s'ils proviennent du site même ou de ses alentours. Enfin, six espèces supplémentaires ont été identifiées : l'Hyène des cavernes (Crocuta crocuta spelaea), le Lion des cavernes (Panthera leo spelaea), le Loup (Canis lupus) et un Renard à déterminer spécifiquement par une étude ostéométrique (Vulpes vulpes ou Alopex lagopus). Les petits mustélidés sont très rares puisque seuls 4 restes (2 hémi-mandibules, 1 crâne complet de grande taille -couche VII- et 1 fragment de coxal) ont été attribués au Putois (Mustela (putorius) putorius), tandis que le Blaireau (Meles meles) n'est représenté que par un humérus en couche III. Ces six espèces sont représentées par 193 restes, représentant $7 \%$ des Carnivores et presque $5 \%$ du NRD.

Le calcul du NMI de fréquence (tabl. 2), effectué en prenant en compte la totalité du matériel faunique de chaque espèce, montre qu'Ursus arctos domine nettement le matériel de Regourdou, non seulement en nombre de restes, mais également en individus potentiels (NMI calculé à partir des vestiges les plus représentés, soit les premières molaires inférieures pour les adultes). En effet, sur les 94 animaux minimum présents sur le site, 36 seraient des ours bruns, soit environ $71 \%$ des Carnivores et près de $40 \%(38,3)$ de la grande faune.

\section{3 - Etude archéozoologique et taphonomique}

Les résultats présentés dans cette partie sont centrés sur les Carnivores, principalement les Ursidés. En effet, la conservation de la grande majorité de leurs vestiges au MNP a permis l'analyse de leurs restes, contrairement au matériel des Herbivores, dont une grande partie n'a été retrouvée que récemment dans les réserves du Musée de Regourdou (Cavanhié 2007).

De par le faible nombre de restes les représentant, les Mustélidés (Blaireau et Putois) n'ont pas été intégrés dans cette étude. Les autres Carnivores non Ursidés sont représentés par peu d'individus (un adulte et un juvénile pour l'Hyène des cavernes, deux adultes et un juvénile pour le Loup et le Lion des cavernes, quatre renards adultes) ainsi que par un faible nombre de vestiges (NR 188). Bien que les éléments squelettiques isolés les plus représentés soient les vertèbres (NR 44, soit 23,4 \%), le « crânien » (dents isolées et mandibules particulièrement) domine avec 65 restes, représentant plus d'un tiers $(34,6 \%)$ du matériel des Carnivores non ursidés, le " thoracique " ( 2 côtes +44 vertèbres) n'en constituant qu'un peu moins d'un quart $(24,5 \%)$. Les os des membres sont peu représentés : la « patte avant " ne représente que 13,3\% avec 25 restes ( 3 fragments de radius, 1 d'ulna et 3 métacarpiens d'Hyène ; 2 fragments d'ulnas de Loup ; 2 humérus de Renard ; 1 radius, 1 ulna, 1 carpien et 1 métacarpien de Lion), la « patte arrière » 16,5\% (NR $31: 2$ fragments de fémur et 1 de fibula pour l'Hyène ; 1 métatarsien et 1 tibia de juvénile pour le Loup ; 1 coxal et 1 tibia pour le Renard ; 2 fragments de fibula, 5 métatarsiens et 10 tarsiens de Lion) et les "sésamoïdes " (sésamoïdes, phalanges et métapodes) $11,2 \%$ avec 1 sésamoïde d'Hyène, 3 métapodes, 12 phalanges et 5 sésamoïdes de Lion.

\subsection{1 - Représentation squelettique de l'ours}

Une première étude taphonomique avait mis en évidence une répartition spatiale différentielle (squelette thoracique principalement hors structure, os des membres majoritairement à l'intérieur de celles-ci), interprétée comme résultant d'un dépeçage des ours sur place (Bonifay M.F. 1989 - p. 46). Le dépôt, à l'intérieur des structures, de dépouilles encore munies des extrémités osseuses des pattes, en avait été proposé comme hypothèse (Bonifay E. 2002 p. 252-253).

L'étude de la représentation squelettique s'est donc basée sur les 1830 restes d'ours (sur 2560, soit plus de $67 \%$ ) pour lesquels la localisation est connue. Les couches et structures ayant livré trop peu de restes ont été exclues de cette étude (deux restes pour les couches I et VIII ; sept pour la couche VIIB ; un seul pour le pierrier VD).

Si elle n'est pas complètement identique, la représentation squelettique pour le matériel d'Ursus arctos découvert hors structure (tabl. 5) semble cependant à peu près homogène dans la majorité des couches (II à V). La moyenne 1 montre que les éléments les plus représentés sont ceux de la catégorie "sésamoïdes ", qui constituent plus d'un tiers (32\%) du matériel faunique contenu dans les couches. Les autres portions squelettiques semblent également réparties, leur pourcentage variant entre 14 et $19 \%$, avec une légère prédominance des membres. Seules deux couches montrent des représentations squelettiques différentes : sous-représentation du squelette thoracique en couche $\mathrm{VI}$ et sous-représentation des éléments de la " patte avant » dans la couche VII. Néanmoins, ces observations sont fortement atténuées par le faible nombre de vestiges contenus dans ces couches, qui gêne la représentativité de l'échantillon. 


\begin{tabular}{|c|c|c|c|c|c|c|}
\hline & CRA & THORA & PATTE AV. & PATTE AR. & SESAM. & Extr. os. \\
\hline $\begin{array}{c}\text { II } \\
\text { (NR 83) }\end{array}$ & 17 & 12 & 18 & 23 & 30 & $\begin{array}{c}46 \\
(10+6) \\
\end{array}$ \\
\hline $\begin{array}{c}\text { III } \\
\text { (112) }\end{array}$ & 22 & 14 & 18 & 19 & 27 & $\begin{array}{c}44 \\
(10+7)\end{array}$ \\
\hline $\begin{array}{c}\text { IV } \\
\text { (276) }\end{array}$ & 19 & 16 & 17 & 17 & 30 & $\begin{array}{c}56 \\
(13+13) \\
\end{array}$ \\
\hline $\begin{array}{c}V \\
(194)\end{array}$ & 16 & 12 & 26 & 16 & 29 & $\begin{array}{c}57 \\
(19+9)\end{array}$ \\
\hline $\begin{array}{c}\text { VI } \\
(35)\end{array}$ & 11 & 9 & 17 & 20 & 43 & $\begin{array}{c}74 \\
(14+17)\end{array}$ \\
\hline $\begin{array}{l}\text { VII } \\
\text { (21) }\end{array}$ & 19 & 19 & 10 & 24 & 29 & $\begin{array}{c}48 \\
(10+10)\end{array}$ \\
\hline $\begin{array}{l}\text { VIIA } \\
\text { (47) }\end{array}$ & 6 & 15 & 30 & 15 & 34 & $\begin{array}{c}51 \\
(13+4)\end{array}$ \\
\hline $\begin{array}{c}\text { Moyenne } 1 \\
\text { couches }\end{array}$ & 16 & 14 & 19 & 19 & 32 & 54 \\
\hline $\begin{array}{l}\text { IIIA } \\
\text { (12) }\end{array}$ & 0 & 8 & 17 & 33 & 42 & $\begin{array}{c}83 \\
(8+33)\end{array}$ \\
\hline $\begin{array}{l}\text { IVA } \\
\text { (283) }\end{array}$ & 24 & 12 & 16 & 17 & 30 & $\begin{array}{c}49 \\
(10+9)\end{array}$ \\
\hline $\begin{array}{l}\text { IVB } \\
(250)\end{array}$ & 24 & 6 & 21 & 19 & 30 & $\begin{array}{c}54 \\
(11+13)\end{array}$ \\
\hline $\begin{array}{l}\text { IVC } \\
(253)\end{array}$ & 20 & 11 & 15 & 21 & 33 & $\begin{array}{c}55 \\
(11+11)\end{array}$ \\
\hline $\begin{array}{l}\text { IVD } \\
\text { (73) }\end{array}$ & 25 & 23 & 12 & 23 & 16 & $\begin{array}{c}25 \\
(1+7)\end{array}$ \\
\hline $\begin{array}{l}\text { VA } \\
\text { (16) }\end{array}$ & 6 & 0 & 56 & 0 & 38 & $\begin{array}{c}81 \\
(44+0) \\
(4)\end{array}$ \\
\hline $\begin{array}{l}\text { VB } \\
\text { (24) }\end{array}$ & 4 & 8 & 17 & 42 & 29 & $\begin{array}{c}67 \\
(4+33)\end{array}$ \\
\hline $\begin{array}{l}\text { VC } \\
(38)\end{array}$ & 8 & 44 & 23 & 5 & 18 & $\begin{array}{c}46 \\
(21+0)\end{array}$ \\
\hline $\begin{array}{c}\text { TOTAL } \\
(2560)\end{array}$ & 19 & 27 & 16 & 15 & 23 & $\begin{array}{c}40 \\
(22+20)\end{array}$ \\
\hline $\begin{array}{l}\text { Moyenne 2 } \\
\text { structures }\end{array}$ & 14 & 14 & 22 & 20 & 30 & 54 \\
\hline Moyenne 1+2 & 15 & 17 & 20 & 19 & 29 & 52 \\
\hline
\end{tabular}

Tableau 5 -

Représentation squelettique (en \%) d'Ursus arctos, par portion squelettique.

Table 5 - Skeletal presentation (in percentage) of Ursus arctos, by skeletal portion.
La répartition squelettique à l'intérieur des structures (tabl. 5) semble plus hétérogène, probablement à cause des grandes différences dans le nombre de restes découverts (12 à 256). Néanmoins, malgré ces écarts, la représentation des portions squelettiques semble très proche de celle observée à l'intérieur des couches : ici aussi, les " sésamoïdes » représentent le tiers du matériel, suivis par les pattes $(22 \%$ pour le membre antérieur, $20 \%$ pour le postérieur), puis par le squelette crânien et thoracique (14\% respectivement).

Ce deuxième examen du matériel semble montrer une représentation quasiment identique des extrémités osseuses des pattes, que ce soit dans les couches ou dans les structures. Ainsi, ces éléments des pattes se retrouvent de façon identique (54\%) que ce soit hors ou dans structures.
Si l'on observe la représentation complète des " patte avant » et « patte arrière » des Ursidés à l'intérieur de la couche IV (dans et hors structures), couche pour laquelle cette hypothèse d'une représentation squelettique avait été formulée, une sur-représentation des métacarpiens et carpiens ainsi que des métatarsiens et tarsiens est en effet nettement visible ; néanmoins, elle pourrait ne refléter que la composition originelle du squelette, ces éléments étant naturellement très nombreux dans un squelette d'Ursidé. De plus, proportionnellement, les éléments des extrémités des pattes sont plus nombreux à l'intérieur de la couche IV que dans les structures. Enfin, la présence d'os des membres ne serait pas explicable dans l'hypothèse d'un dépôt de fourrure.

De manière générale (tabl. 6), le squelette crânien est nettement dominé par les dents isolées, et les extrémités 


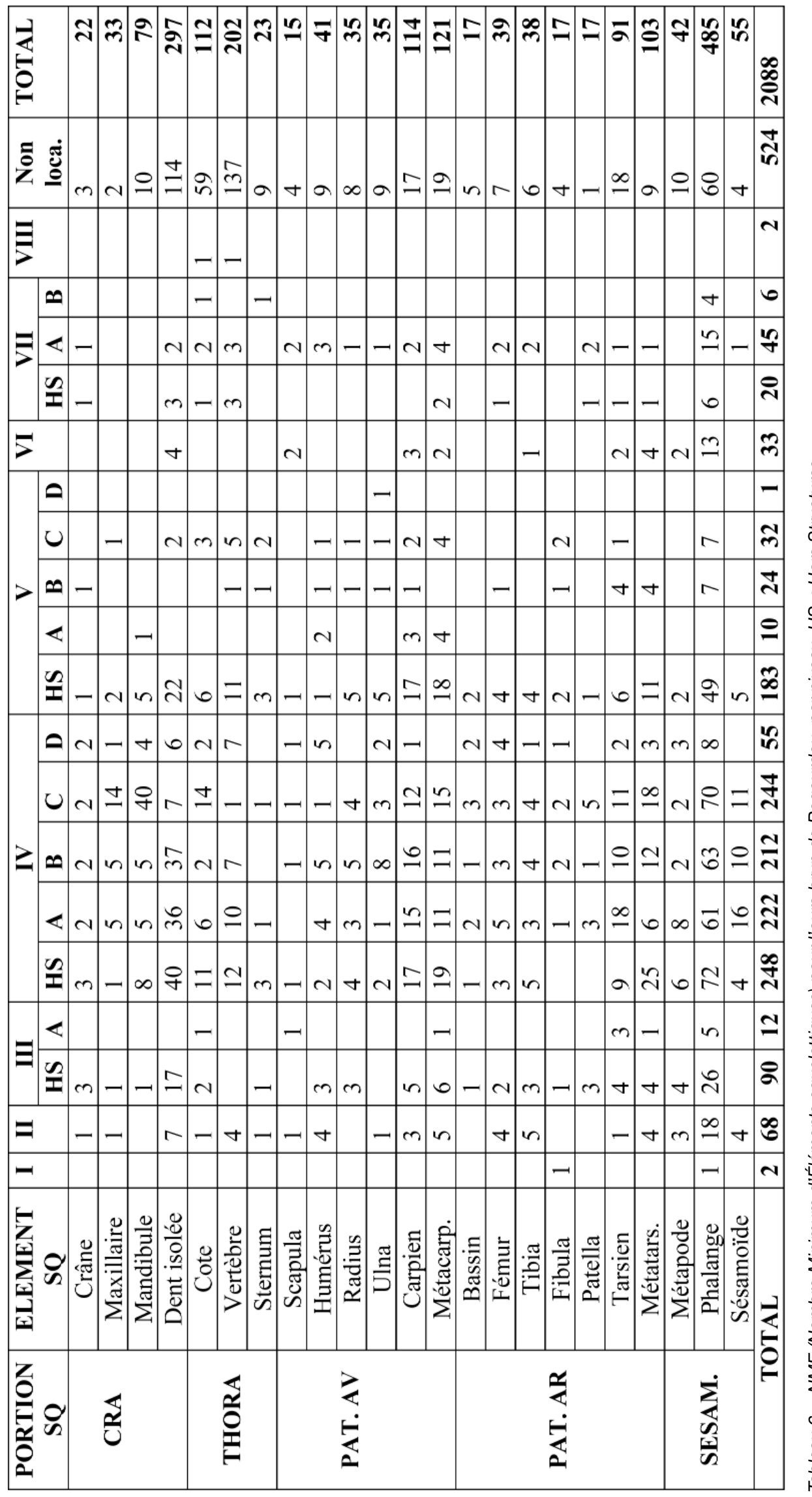

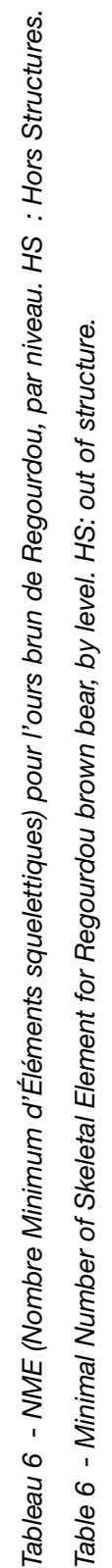


osseuses des pattes (catégorie " sésamoïdes ") par les phalanges. Les os des membres sont représentés sur le gisement par un nombre de restes sensiblement équivalent. Le squelette thoracique montre une très forte représentation des vertèbres. La sous-représentation des côtes, habituelle sur un site archéologique étant donné la fragilité de ces éléments osseux, est compensée par le nombre important de fragments non localisés, qui montrent des fractures fraîches.

Les éléments les plus fréquents sont les dents et les éléments du carpe et du tarse, qui constituent les éléments osseux les plus représentés quantitativement naturellement mais aussi les plus résistants aux divers phénomènes taphonomiques de par leur densité osseuse. Tous les éléments du squelette d'Ursus arctos, même les plus petits (carpiens et tarsiens), sont présents sur le gisement de Regourdou, et en nombre sensiblement équivalent pour les éléments pairs (os longs, dents, etc.).

\subsection{2 - Structure démographique de la population ursine : classes d'âge et sex-ratio}

La courbe de mortalité constituée grâce à l'étude des différents éléments dentaires (cf. III-1.A-Méthode) (fig. 5) montre une sur-représentation des juvéniles, les classes d'âges suivantes déclinant de façon continue.

L'établissement du sex-ratio des Ursidés de Regourdou a été gêné par de nombreux problèmes, notamment le très faible nombre d'os longs entiers et donc mesurables (tabl. 7). Malgré ces limites, il a pu être estimé, grâce à l'étude ostéométrique des dents (fig. 6). En dépit du nombre important de canines, inférieures (NR : 20) et supérieures (NR : 26), peu d'entre elles ont pu être étudiées du point de vue du dimorphisme sexuel. En effet, outre des cassures empêchant la prise de mesure sur certaines, un grand nombre montrent une racine ouverte, caractéristique des sub-adultes : elles n'ont donc pas été intégrées dans cette étude afin de ne pas fausser l'estimation du sex-ratio des Ursidés de Regourdou. II est toutefois intéressant de noter que dans la quasi-totalité des cas, les canines montrant une racine ouverte semblent, par leurs dimensions, appartenir à des ours juvéniles mâles. Les mesures obtenues ont ensuite été comparées avec les données publiées (Koby 1949 - p. 681,682 ; Altuna 1973 ; Torres Pérez Hidalgo 1988). Le matériel dentaire étudié, à l'exception des canines supérieures, semble montrer une sur-représentation des mâles qui constituent, d'après les mesures sur les différents éléments dentaires, jusqu'à $71 \%$ de la population d'Ursidés de Regourdou.

L'étude taphonomique a été gênée par les nombreuses altérations récentes, contemporaines ou postérieures aux fouilles, présentes sur le matériel. Ces altérations prennent la forme de fractures fraîches (900 restes, soit $23 \%$ du $\mathrm{NRD}$ ) et, moins fréquemment, de stries récentes (NR : 22, soit $0,5 \%$ du NRD) : les déménagements successifs vécus par le matériel faunique en sont la cause la plus probable. L'émiettement du matériel et la disparition de la surface corticale pourraient être responsables d'une dispari-

\begin{tabular}{|c|c|c|c|c|c|c|c|c|}
\hline & & NR & C & P+ & D+ & Ep & Ed & Fd \\
\hline \multirow{2}{*}{ HUMERUS } & A & 43 & 3 & 2 & 16 & 6 & 7 & 9 \\
\cline { 2 - 9 } & SA & 14 & 1 & 1 & 6 & 7 & & 1 \\
\hline \multirow{2}{*}{ RADIUS } & A & 28 & 2 & 11 & 4 & 1 & & 10 \\
\cline { 2 - 10 } & SA & 16 & & 1 & 2 & & 12 & 1 \\
\hline \multirow{2}{*}{ ULNA } & A & 36 & 3 & 17 & 7 & 3 & 1 & 5 \\
\cline { 2 - 9 } & SA & 4 & 1 & 2 & & 1 & & \\
\hline \multirow{2}{*}{ FEMUR } & A & 35 & 3 & 6 & 4 & 7 & 4 & 11 \\
\cline { 2 - 9 } & SA & 19 & 4 & 3 & & 5 & 6 & 1 \\
\hline \multirow{2}{*}{ TIBIA } & A & 24 & 4 & 4 & 5 & 5 & 2 & 4 \\
\cline { 2 - 9 } & SA & 23 & 1 & 3 & 2 & 6 & 8 & 2 \\
\hline \multirow{2}{*}{ FIBULA } & A & 16 & 1 & 5 & & 6 & 1 & 3 \\
\cline { 2 - 8 } & SA & 2 & 1 & & & & & 1 \\
\hline
\end{tabular}

Tableau 7 - Distribution des portions osseuses pour les os longs d'Ursus arctos de Regourdou. A : Adulte ; SA : Subadulte. $C$ : Complet ; $P_{+}$: Articulation proximale + diaphyse ; $D_{+}:$Articulation distale + diaphyse ; Ep : Epiphyse proximale seule ; Ed : Epiphyse distale seule ; Fd : Fragment diaphysaire.

Table 7 - Regourdou's fragment type distribution of Ursus arctos long bones. A : Adult ; SA : Sub-adult. C : Complet ; $P_{+}$: Proximal end + shaft $; D_{+}$: Distal end + shaft ; Ep : Proximal epiphysis only ; Ed : Distal epiphysis only ; Fd : Shaft fragment.

tion quantitative de certaines altérations taphonomiques. Au niveau des altérations anciennes subies par le matériel, plusieurs agents, abiotiques et naturels, sont impliqués. Néanmoins, l'action des agents abiotiques étant très peu représentée (concrétionnement : 0,15\% ; dissolution $0,3 \%$ et charriage : $0,7 \%$ du NRT), seules les altérations dues à des agents biologiques seront traitées ici, à l'exception de l'action des rongeurs, visible sur 6 pièces (soit $0,2 \%$ NRT) dispersées spatialement.

\subsection{3 - Traces anthropiques sur l'ensemble des restes osseux}

La totalité du matériel osseux de Regourdou conservé au Musée National de Préhistoire des Eyzies-de-Tayac (Dordogne) a été examinée très attentivement afin d'identifier d'éventuelles traces anthropiques.

Pour les Herbivores, seuls quatre ossements, d'individus adultes uniquement, ont livré des stries de découpe : une phalange (remanié), un humérus (couche VII) et une vertèbre dorsale de cerf (VIIIA) et un radius de cheval (couche II). Uniquement à l'intérieur de l'ensemble supérieur de la stratigraphie (couche II), une fracturation anthropique (récupération de la moelle ?) a également été remarquée : elle ne concerne que les ossements de rennes et est visible sur 27 pièces ( 5 humérus, 5 radius, 10 métacarpes, 3 tibias et 4 métatarses) sur 282 , soit près de $10 \%$.

Pour les Carnivores, seuls les Ursidés semblent avoir été l'objet d'une potentielle exploitation humaine. Ainsi, une extrémité distale de tibia gauche (Reg 41059 G5-70), appartenant à un individu adulte et provenant de la couche IV, porte cinq stries groupées, présentes sur la face 

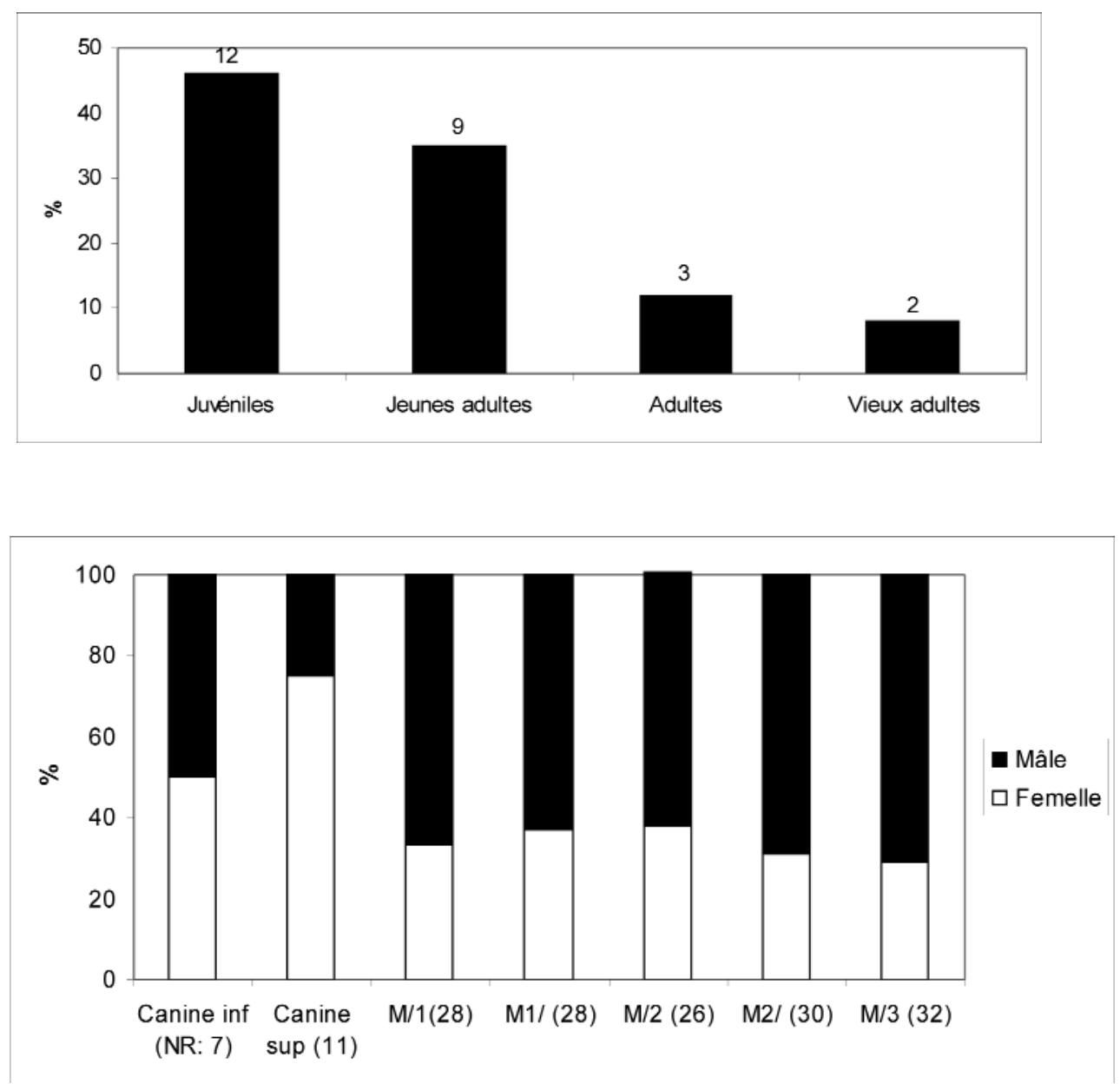

Figure 5 - Représentation (en \%) des classes d'âge pour les Ursidés de Regourdou (en haut, pour chaque catégorie : NMI).

Figure 5 - Age groups (in \%) of Regourdou brown bears (above, for each category: NMI).
Figure 6 - Sex-ratio des Ursidés de Regourdou, d'après les différents éléments dentaires (NR).

Figure 6 - Sex-ratio of Regourdou brown bears, according to the different dental elements (NR) médiale, à proximité d'une sixième strie, plus discrète (fig. 7). Deux fragments diaphysaires de côtes d'adultes portent également des stries. Sur la surface de la première (Reg 43417 M5 44), deux groupes de stries, relativement superficielles, sont visibles : le premier comprend quatre stries longitudinales, tandis que le deuxième regroupe trois stries quasiment perpendiculaires à l'axe de la côte. La deuxième côte (Reg $7 A$ 801) porte cinq stries très profondes, transverses et parallèles entre elles, bien qu'espacées (fig. 8). Toutes ces stries pourraient être attribuées à une activité de dépouillement ou de décharnement.

\subsection{4 - Action des Carnivores sur l'ensemble des restes osseux}

L'action des Carnivores est visible sur 140 pièces (soit $3,5 \%$ du NRD), dont 134 (soit $96 \%$ ) appartiennent à l'Ours, quatre à des Herbivores (3\%) et deux à un Carnivore non Ursidé, l'Hyène ( $1 \%)$, soit près de $5 \%$ du matériel total (NRT). Elle est particulièrement concentrée sur les 61 os longs d'ours (44\% du matériel affecté par l'action des Carnivores), puisqu'elle concerne $36 \%$ des humérus, $38 \%$ des radius, $29 \%$ des ulnas, $21 \%$ des fémurs et $23 \%$ des tibias, soit un tiers des os longs en moyenne. De plus, alors que les autres pièces (os du méta- pode, vertèbres, côtes, etc.) montrent un rongement superficiel (bords très légèrement crénelés), les os longs témoignent de rongements d'une intensité très variable : de très légère, l'action des Carnivores peut aller jusqu'à la destruction totale des épiphyses, de la diaphyse, voire la destruction quasi-totale de l'os. En effet, l'action des Carnivores se manifeste par un écrasement des parties spongieuses des épiphyses, entraînant leur disparition partielle ou totale ; il ne subsiste alors de l'os qu'un fragment diaphysaire plus ou moins long selon l'intensité de rongement (pl. 1).

Cette action se caractérise également par la présence sur les os de plusieurs stigmates: des entailles, de profondeur variable, ont été repérées sur 9 des 61 os longs (soit moins de $15 \%$ ), tandis que des petits enfoncements, peu profonds et de faible taille, sont présents sur $46 \%$ de ce matériel (NR : 28), au niveau de l'os compact. Enfin, un début d'écrasement de la surface corticale est visible sur deux ossements, représentant environ $3 \%$.

Ces observations ont été comparées avec différents travaux caractérisant les dommages osseux dus aux Carnivores connus pour leur rôle dans les modifications osseuses (Dominguez-Rodrigo et Piqueras 2003), 


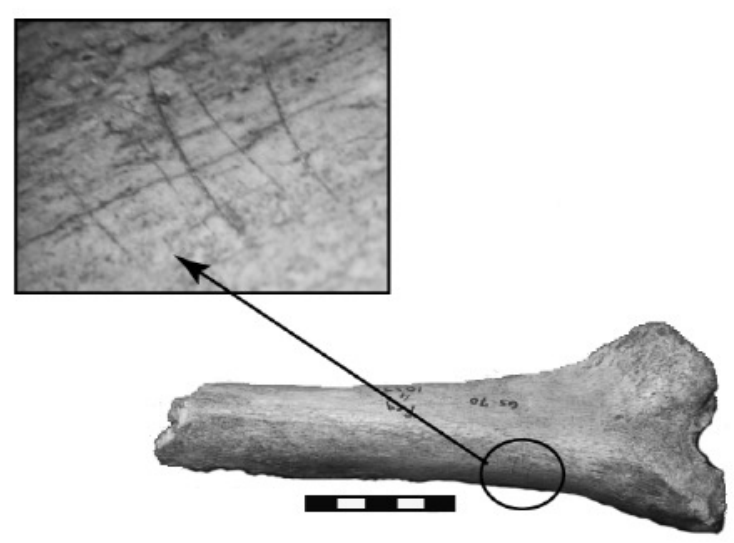

Figure 7 - Extrémité distale de tibia d'ours brun portant des stries anthropiques (grossissement à la loupe binoculaire x10).

Figure 7 - Distal epiphysis of brown bear's tibia with cut marks (magnification in the binocular magnifier X10).

notamment l'Hyène (Avery et al. 1984 ; Harle 1882 ; Haynes 1980 ; Henschel et Tilson 1988 ; Hill 1989 ; Klein 1975 ; Klein et al. 1991 ; Skinner et al. 1986) et le Loup (Castel 2004). Aucune de ces descriptions n'était comparable aux altérations visibles sur le matériel de Regourdou. Au contraire, les dommages visibles sur celui-ci ressemblent aux rares descriptions publiées des dommages osseux causés par des ours, dans lesquelles l'émoussement des bords et des renflements de l'os et le broiement des surfaces compactes, parfois accompagnés d'éraflures sans orientation et de rainures parallèles (pouvant rappeler l'action des rongeurs), sont définis comme caractéristiques de l'action des Ursidés (Haynes 1983 - p.169). Le matériel osseux provenant du site à Ours des cavernes de La Balme à Collomb (Savoie) (étude réalisée par Lolliot et Philippe 2004) présente des similitudes avec le matériel issu des fouilles de Regourdou : outre un émoussement des parties spongieuses qui affecte principalement les extrémités des os longs, qualifié de « rognage » (fig. 9), les auteurs décrivent des "rainures ", " entailles plus ou moins profondes et allongées, quelquefois parallèles ", situées essentiellement sur les diaphyses des os longs, les bords des scapulas et autres os plats, des « pits ", «petits enfoncements au niveau de l'os compact, peu profonds et de faible taille ", et des « punctures », enfoncements de l'os sous la pression des dents, pour la plupart au niveau des extrémités des os longs, plus tendres (Lolliot et Philippe 2004 - p. 117-119). Tous ces stigmates correspondent totalement à ceux décrits pour Regourdou (à l'exception peut-être des « punctures » qui, sur Regourdou, pourraient correspondre à une première étape, la pression de la dent n'ayant pas été assez forte pour traverser la surface corticale). En l'absence de trace de digestion, ces traces de charognage ont été attribuées aux ours euxmêmes, leur comportement de nécrophagie, voire de cannibalisme, à la sortie de l'hibernation étant connu (Lolliot et Philippe 2004 - p.120).

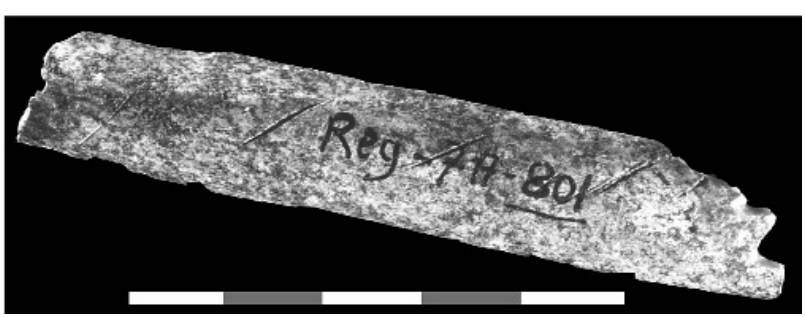

Figure 8 - Fragment diaphysaire de côte d'ours brun portant des stries anthropiques. Photographie : Ph. Jugie (MNP).

Figure 8 - Brown bear's rib fragment with cut marks. Photography : Ph. Jugie (MNP).

Les observations effectuées sur le matériel de Regourdou sont similaires à celles d'études taphonomiques consacrées aux dégâts causés par les ours eux-mêmes, ainsi qu'aux constatations réalisées sur le matériel faunique du Mont Ventoux, site dans lequel les seuls grands Carnivores représentés sont des ours bruns, seuls à même d'avoir réalisé les altérations présentes sur les ossements d'ours (Ph. Fosse, com. pers.) : il semblerait que les ours de Regourdou soient les responsables de ces destructions osseuses. Bien qu'il soit aujourd'hui attesté que les ours (Ursus spelaeus comme arctos) constituent les principaux responsables de la désarticulation des restes osseux d'ours morts en hibernation (Pinto Llona et Andrews 2004 p.163), il serait cependant nécessaire de comparer le matériel de Regourdou avec un matériel, fossile ou expérimental, affecté avec certitude par des Ursidés, afin de confirmer cette interprétation.

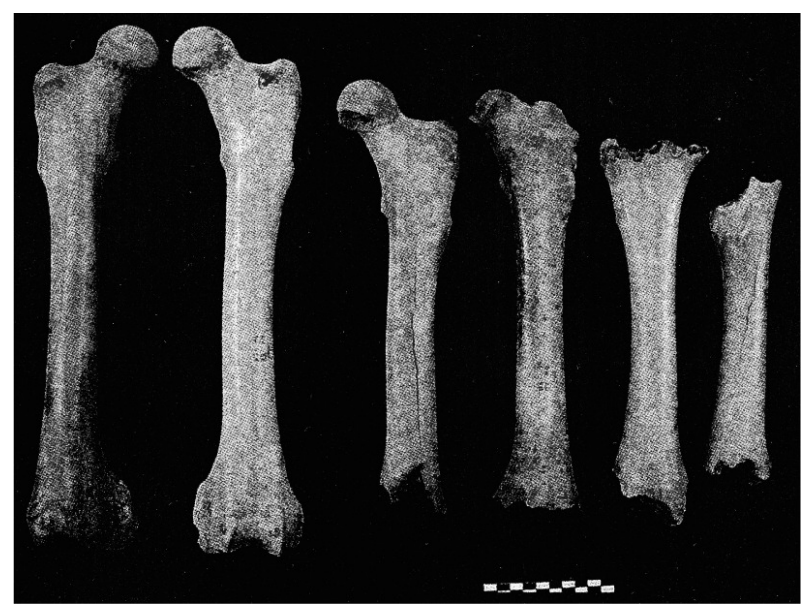

Figure 9 - Fémurs d'ours des cavernes de La Balme à Collomb, présentant différents stades de charognage (Lolliot et Philippe 2004 - p.116, photo 1).

Figure 9 - Femurs of cave bears of La Balme à Collomb, with different stages of charognage (Lolliot et Philippe 2004 - p.116, photo 1). 


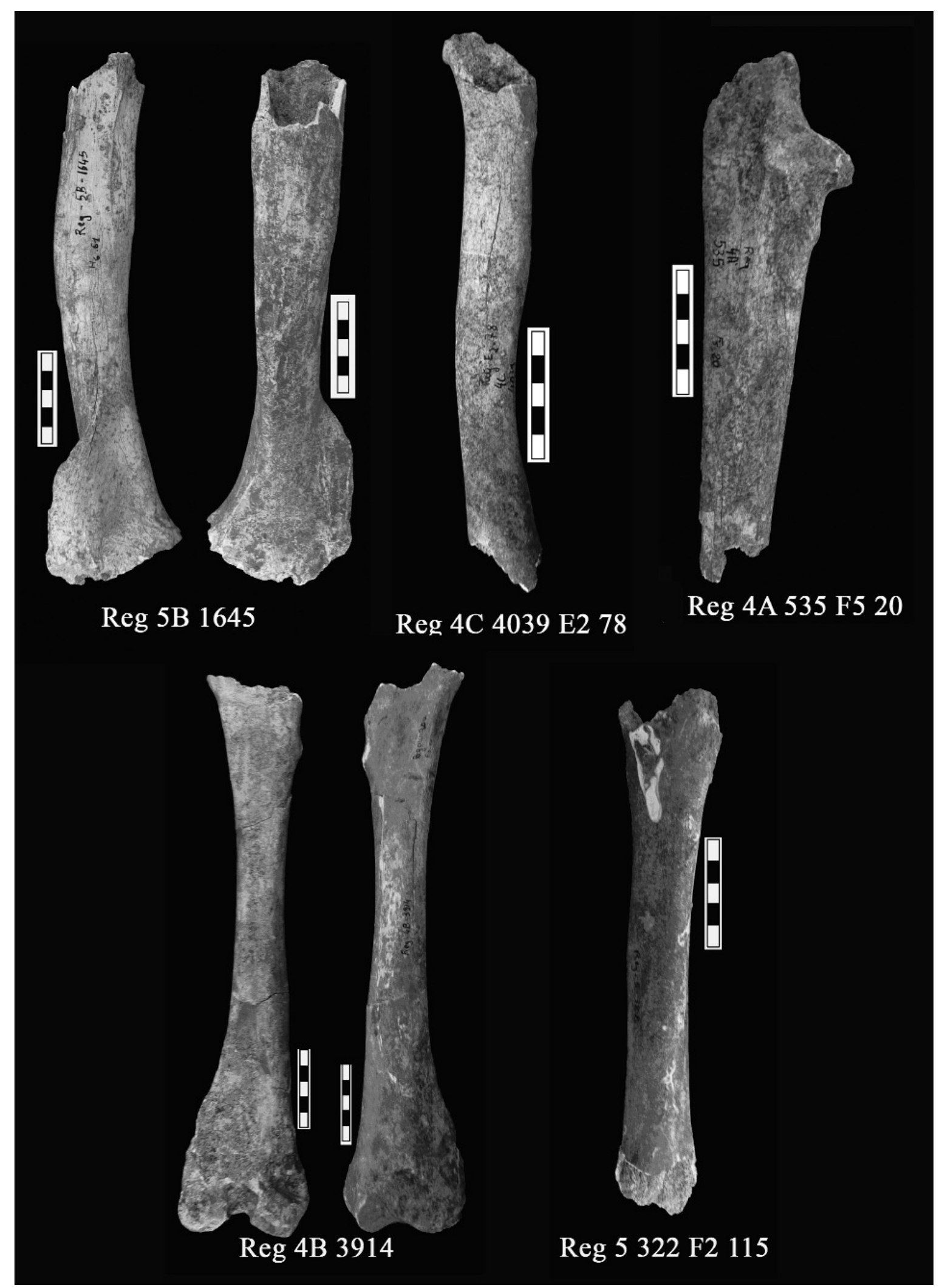

Planche I - Os longs d'ours bruns présentant différents stades de charognage : humérus (Reg 5B 1645), radius (Reg 4C 4039 E2 78), ulna (Reg 4A 535 F5 20), fémur (Reg 4B 3914) et tibia (Reg 5322 F2 115).

Planche I - Different stages of charognage on long bones of Regourdou brown bears : humerus (Reg 5B 1645), radius (Reg 4C 4039 E2 78), ulna (Reg 4A 535 F5 20), fémur (Reg 4B 3914) et tibia (Reg 5322 F2 115). 


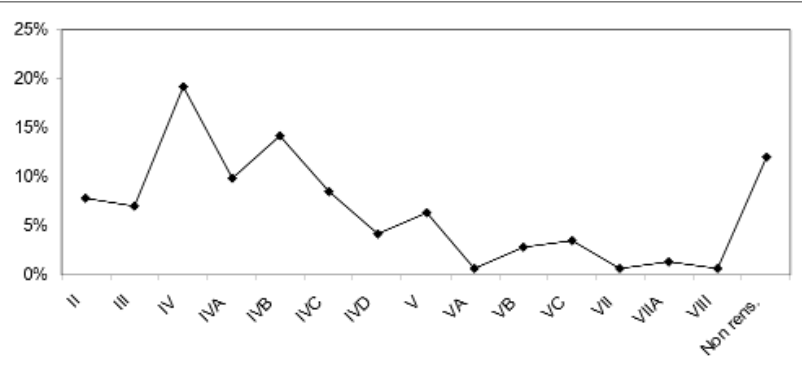

Figure 10 - Répartition par couche des restes présentant une activité des Carnivores (NR : 140).

Figure 10 - Repartition by level of faunal remains with a carnivorous' activity.

L'étude de la répartition spatiale des 140 restes osseux altérés par des Carnivores a été possible (fig. 10), $12 \%$ seulement de ce matériel n'étant pas renseigné spatialement. Une concentration particulièrement forte est visible dans la couche IV, qui contient $19 \%$ des restes hors structures, et $56 \%$ en additionnant les ossements retrouvés dans et hors structure.

\section{4 - Conclusions}

Les études archéozoologiques et taphonomiques ont donc permis de mettre en évidence plusieurs caractéristiques propres au matériel faunique des Ursidés de Regourdou :

- une prédominance sur les autres espèces présentes, que ce soit en termes de Nombre de Restes (près de $65 \%$ du NRD, plus de $92 \%$ du matériel des Carnivores) ou de Nombre Minimum d'Individus (71\% des Carnivores, $40 \%$ de la grande faune) ;

- une représentation squelettique équilibrée (présence de tous les éléments osseux, et en nombre sensiblement égal pour les os pairs -os longs, carpiens et tarsiens-) ;

- une population dominée par les mâles et les juvéniles, les autres classes d'âge décroissant régulièrement ;
- un matériel présentant une importante activité des Carnivores (sûrement des ours eux-mêmes), contrairement à l'activité anthropique très faiblement représentée (stries de boucherie présentes sur 7 ossements, dont 3 d'ours).

\subsection{1 - Sur la présence des restes de grands mammifères, des ours notamment}

Les ours bruns présents sur le site de Regourdou sont représentés par la totalité des éléments osseux, en nombre sensiblement équivalent selon leur latéralisation, pour les éléments du carpe et du tarse comme pour les os longs. Equilibrée, cette représentation squelettique montre une légère sur-représentation des os communs au carpe et au tarse (les sésamoïdes et phalanges, ainsi que les métapodes) et des restes crâniens (dents isolées en particulier). La comparaison la plus probante a été celle réalisée entre le Nombre Minimum d'Eléments (NME) présents naturellement dans une carcasse d'ours et le NME moyen calculé pour les ours de Regourdou, à l'intérieur des couches, à l'intérieur des structures et en associant couches et structures (fig. 11). En effet, hormis une sous-représentation du squelette thoracique sur le site explicable d'une part par la fragilité des côtes, pouvant entraîner leur disparition prématurée, et d'autre part, par le nombre important de côtes (NR : 59/112) et vertèbres (NR : 137/202) non localisées stratigraphiquement, le rang d'importance de la plupart des portions squelettiques semble identique (prédominance des " sésamoïdes " suivi des éléments du squelette crânien ; grande proximité des valeurs pour les membres antérieurs et postérieurs).

La représentation squelettique équilibrée observée pourrait donc être le résultat d'une mort des animaux in situ ; toutefois, elle pourrait également résulter du transport par les hommes des carcasses entières des plantigrades sur le site, explication qui semble être celle donnée par $E$. et M.F. Bonifay (Bonifay M.F. 1989 ; Bonifay E. 2002).

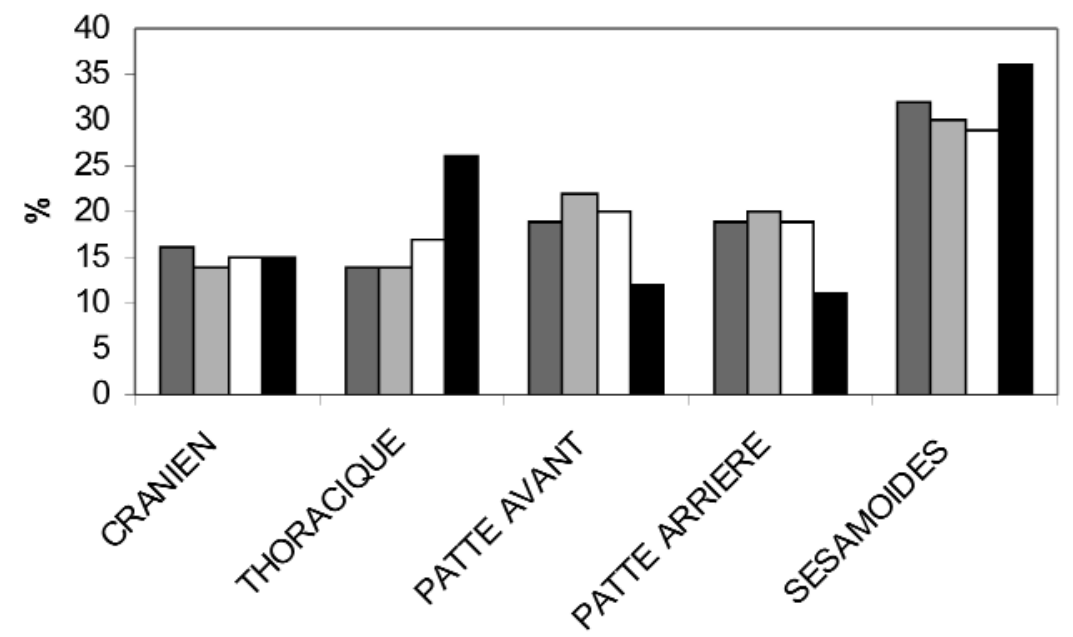
$\square$ Moyenne NME (couches Regourdou)

$\square$ Moyenne NME (structures Regourdou)

口Moyenne NME (couches+structures Regourdou)

NME d'un squelette d'ours

Figure 11 - Comparaison entre le NME calculé pour les ours de Regourdou et le NME observé naturellement dans un squelette d'ours brun.

Figure 11 - Comparison between MNE calculated for the Regourdou bears and natural MNE of a brown bear skeleton. 
Néanmoins, cette théorie contraste avec les observations taphonomiques réalisées sur le matériel, qui ont mis en évidence une altération relativement forte d'environ un tiers des os longs d'ours. Si ces destructions osseuses sont dues, comme cela est fortement présumé, aux Ursidés, elles montreraient une occupation plus ou moins longue du site par les ours et excluraient la possibilité qu'ils aient été amenés à l'état de carcasses.

Cette hypothèse pourrait être confirmée par le nombre relativement important d'ours potentiellement présents (NMI 36) sur le site. L'étude de la structure démographique (sex-ratio et classes d'âges) de populations préhistoriques d'Ours des cavernes et de Deninger a permis de déterminer une fréquentation différente des tanières, certaines grottes étant fréquentées majoritairement par les ourses suitées (“ pouponnières "), d'autres par les mâles et d'autres, enfin, montrant une occupation mixte (Fosse et al. 2002 - p. 84). A Regourdou, le sex-ratio pourrait indiquer une présence majoritaire des mâles.

Cette hypothèse d'une mortalité naturelle des ours semble également confirmée par la structure démographique : au sein d'une population d'ours, les adultes sont minoritaires, contrairement aux individus jeunes et âgés qui sont majoritaires. La courbe établie pour les ours de Regourdou montre au contraire une diminution régulière du nombre de représentants pour les classes d'âges suivant les juvéniles, qui pourrait refléter une mortalité catastrophique (Stiner 1990).

Les observations réalisées sur le matériel de Regourdou pourraient donc indiquer une mortalité naturelle des ours pendant l'hibernation et, pour la majorité des individus, dans leur première année, moment où ils sont les plus vulnérables. Le nombre important de jeunes morts durant leur premier hiver (entre 8 et 12 mois, soit entre septembre et janvier, les oursons naissant normalement vers janvier) semble également confirmer cette hypothèse. L'absence des très jeunes oursons (0-4 mois) n'est peut-être que le reflet d'une mauvaise conservation, conservation différentielle in situ d'une part, les ossements et dents d'immatures se conservant moins bien que les restes d'adultes et disparition récente d'une partie du matériel due aux déménagements successifs d'autre part. E. Bonifay parle par exemple de quelques bourgeons dentaires d'un très jeune ourson découverts dans la fosse IIIA (Bonifay E. 2002 p. 250) qui n'ont pu être retrouvés dans le matériel. II est donc tout à fait envisageable qu'une disparition d'une partie du matériel soit responsable de l'absence des très jeunes oursons dans la représentation en classes d'âges : le site de Regourdou pourrait tout de même avoir servi de pouponnière, comme pourrait le montrer la prédominance des juvéniles (quatre mois à un an).

Ces observations taphonomiques, cumulées à une représentation squelettique équilibrée pour Ursus arctos, sembleraient donc plutôt indiquer une origine naturelle des ours de Regourdou : ils seraient morts in situ, pendant I'hibernation. Néanmoins, la richesse taxonomique du gisement pose problème. En effet, d'après les observations actuelles menées sur leur comportement d'hibernation, aucun ours n'amènerait de carcasse dans sa tanière, afin de ne pas attirer de prédateur pendant qu'il est en position de faiblesse (Stiner 2002 - p.159). Dès lors, comment expliquer la présence des nombreux restes d'herbivores sur le site ? Plusieurs hypothèses peuvent être formulées : origine anthropique (chasse), origine animale (prédation) ou origine naturelle (piège). Le matériel des Herbivores qui a pu être examiné au cours de cette étude ne montre que peu de traces d'agents taphonomiques biologiques: quatre pièces montrent une faible activité des Carnivores et deux une intense activité des rongeurs. Les activités anthropiques sont représentées par des stries de boucherie (cut marks) sur quatre ossements ( 3 de cerf et 1 de cheval) et par une fracturation (recherche de la moelle ?) sur 27 os longs de renne de la couche II. La faiblesse de ces altérations et la nature de leurs supports incitent à penser que le charognage ou la chasse par les Carnivores et/ou les Hommes n'est pas à l'origine de ces accumulations osseuses, hypothèse qui pourrait être confirmée par le faible NMI des Carnivores extra Ursidés (deux loups, quatre renards, deux hyènes et trois lions des cavernes). La dernière hypothèse, celle d'un piégeage naturel, semble privilégiée. Elle correspondrait à la configuration du site : une cheminée naturelle (« puits Constant») (fig.1), probable unique accès du site au Paléolithique, aurait pu jouer le rôle d'aven piège.

Cette hypothèse d'une mortalité naturelle peut également être formulée pour les Carnivores non Ursidés, soit l'Hyène et le Lion des cavernes, le Loup et le Renard. En effet, aucun des ossements qui leur ont été attribués ne porte de stigmate d'une quelconque action anthropique (fragmentation, stries de découpe, etc.). Quant à l'action des Carnivores, elle est plus que limitée (deux ossements d'Hyène présentant un très léger rongement).

\subsection{2 - Activités humaines}

L'hypothèse d'une mortalité naturelle des Ursidés n'exclut pas pour autant l'intervention humaine. Toutefois, celle-ci ne pourrait être que ponctuelle, limitée à une structure (tumulus VIIA) et à la couche IV, définie comme le seul niveau qui pourrait constituer un habitat temporaire (Bonifay E. 1963 - p. 53). La présence humaine y est en effet attestée de manière incontestable par la sépulture néandertalienne. L'interaction Homme/Ours est certifiée dans ce niveau, par la présence de deux des trois ossements d'ours bruns portant des stries de découpe, retrouvés hors structure. Les exemples de boucherie, ethnographiques et expérimentaux, ont démontré qu'un coup donné n'induit pas obligatoirement la création de marques (Gifford-Gonzalez 1989). Néanmoins, le faible nombre de stries retrouvées, associé au nombre élevé d'ossements d'ours présents sur le site ainsi qu'au nombre important d'altérations taphonomiques imputées aux ours euxmêmes, incite à émettre l'hypothèse d'une interaction Homme/Ours ponctuelle, sans qu'il soit possible de déterminer s'il s'agit de chasse ou de charognage occasionnel (récupération de la fourrure, du cuir et/ou de la chair). 
Il est intéressant de noter que la couche contenant la plus forte action anthropique (fracturation pour une probable récupération de la moelle visible sur $10 \%$ des ossements de rennes) est la couche II, soit la couche où le ratio du nombre de restes de Carnivores par rapport à celui des herbivores est le plus faible (0,3\%) (tabl.2).

\subsection{3 - Fonction(s) du site (tabl. 8)}

Cette hypothèse d'une interaction Homme/Ours ponctuelle contraste fortement avec l'interprétation du site telle qu'elle a été donnée par son fouilleur, E. Bonifay, pour qui tous les ossements d'ours présents sur le site étaient d'origine anthropique. Cet antagonisme peut s'expliquer par les éléments pouvant sembler contradictoires, résumés dans le tableau 8, qui ressortent de l'étude du matériel.

Malgré les problèmes de fiabilité sur la localisation exacte du matériel, des différences semblent apparaitre entre les ensembles stratigraphiques supérieur (couches I et II) et inférieur (couche III à VIII) et ce, tout d'abord d'un point de vue géologique, le site ayant cessé de fonctionner en tant que grotte entre les niveaux II et III, suite à l'effondrement du plafond au début du Würm II (Bonifay E. 2002). L'ensemble supérieur a livré l'action anthropique la plus importante quantitativement (fracturation visible sur près de $10 \%$ des os longs de renne de la couche II), à l'intérieur de la couche où la présence des Carnivores, notamment Ursidés, est la plus faible.

Cette observation pourrait permettre d'envisager une origine principalement anthropique des vestiges osseux, bien que l'action des Carnivores, présente sur environ $8 \%$ du matériel faunique de la couche II, implique une fréquentation de ces niveaux par les ours.
Si la fréquentation humaine dans l'ensemble inférieur est attestée de manière incontestable par la sépulture néandertalienne, aucune observation ne semble confirmer l'origine anthropique du matériel osseux. En effet, la faible fréquence des stries de découpe (présentes sur 7 ossements, soit $0,08 \%$ du NRD) contraste avec l'action des Carnivores visible sur 3,5\% des restes déterminés, qui semble prouver une occupation plus ou moins longue du site par les ours, contrastant avec la rareté des stries anthropiques. La répartition squelettique différentielle observée par M.F. Bonifay lors de sa première étude taphonomique d'une partie du matériel n'a pas pu être retrouvée. En effet, la représentation des extrémités osseuses des pattes semble identique, ou quasiment, à l'intérieur des couches comme au sein des structures. L'hypothèse de dépôt de fourrure dans les structures de la couche IV a donc dû être abandonnée. De plus, la représentation squelettique équilibrée pour les Ursidés s'apparente à celle d'animaux morts naturellement (présence de tous les os, même les plus petits), malgré le fait qu'aucune connexion anatomique n'ait été signalée par les fouilleurs. Enfin, l'établissement de classes d'âge révèle une courbe de mortalité catastrophique. De nombreux indices incitent donc à penser une mortalité des Ursidés in situ. Deux scénarios sont alors envisageables : celui d'un piégeage naturel, qui correspondrait à la configuration du site (le " puits Constant ", cheminée large de 2 mètres, étant le seul accès actuellement connu), ou une mort pendant l'hibernation.

Les preuves de l'interaction Homme/Ours à Regourdou sont donc rares, laissant penser que cette relation n'a été que très ponctuelle, limitée au tumulus VIIA et surtout à la couche IV, la couche de la sépulture. Cette couche est également isolée du point de vue de l'industrie lithique car les vestiges qu'elle a livrés se distinguent de ceux des autres couches par un débitage discoïde (A. Turq, com. pers.).

\begin{tabular}{|c|c|c|c|c|c|c|}
\hline \multirow[t]{2}{*}{ Critère observé } & \multirow[t]{2}{*}{ Stratigraphie } & \multirow[t]{2}{*}{ Critère observé } & \multicolumn{3}{|c|}{ Agent taphonomique } & \multirow[t]{2}{*}{ Remarque } \\
\hline & & & Naturel & Ours & Homme & \\
\hline Topographie site & \multirow{4}{*}{$\begin{array}{l}\text { ENSEMBLE SUP } \\
\text { (couche II) } \\
\text { (provenance } \\
\text { incertaine : } \\
23,3 \% \text { du matéricl) }\end{array}$} & & $\mathrm{x}$ & & $\mathrm{X}$ & Plafond grotte ellondré \\
\hline Ratio Carnivores/()ngulés & & 0,3 & $\mathrm{x}$ & & $\mathrm{X}$ & \\
\hline Activité des carnivores & & $8 \%$ env. & $\mathrm{x}$ & $\mathrm{x}$ & & \\
\hline Fracturation anthropique & & $10 \%$ cnv. & & & $\mathrm{X}$ & Os longs de Renne \\
\hline Topographie site & \multirow{8}{*}{$\begin{array}{l}\text { ENSEMBLE INF } \\
\text { (provenance } \\
\text { incertaine : } \\
23,9 \% \text { du matériel) }\end{array}$} & & $\mathrm{x}$ & $\mathrm{x}$ & & grotte I cheminée \\
\hline Ratio Carnivores/Ongulés & & 4,7 (moyenne) & $\mathrm{x}$ & $\mathrm{x}$ & & \\
\hline Présence d'herbivores & & NR: 401, soil $18,8 \%$ & $\mathrm{x}$ & & $\mathrm{X}$ & Diachronie? \\
\hline Activité des carnivores & & $\begin{array}{c}\text { NR: I40, soit } 3,5 \% \\
\text { NRD }\end{array}$ & $\mathrm{x}$ & $x$ & & \\
\hline Traces anthropiques & & $\begin{array}{c}\mathrm{N}: 7, \text { soit } 0,08 \% \\
\text { NRD }\end{array}$ & & & $\mathrm{X}$ & \\
\hline $\begin{array}{c}\text { Représentation squelettique des } \\
\text { Ursidés }\end{array}$ & & & $x$ & $x$ & & Equilibrée \\
\hline Classes d'âge des Ursidés & & & & $x$ & & $\begin{array}{c}\text { Prédominance juvéniles }+ \\
\text { subadultes }\end{array}$ \\
\hline Courbe de mortalité des Ursidés & & & $\mathrm{x}$ & $\mathrm{x}$ & & Courbe catastrophique \\
\hline
\end{tabular}

Tableau 8 - Bilan des observations taphonomiques relevées sur les ossements de Carnivores et d'Ongulés du site de Regourdou.

Table 8 - Synthesis of the taphonomic observations on the Regourdou's Carnivora and Ongulates bones. 


\section{3 - Comparaison avec d'autres sites à ours}

Le but de cette reprise d'étude du matériel faunique de Regourdou est de participer à la discussion autour de l'histoire taphonomique de l'assemblage, en étudiant différents critères diagnostiques permettant éventuellement de mettre en évidence l'origine de ce matériel osseux. La présence en masse d'ours bruns dans un site du Paléolithique moyen a été vu comme un élément important, voire l'élément central, pour l'interprétation du gisement ; aussi était-il important de remettre ce site dans un contexte plus général, de le comparer avec d'autres sites ayant livré des accumulations d'ossements d'ours. Cependant, face à la relative rareté de l'Ours brun dans les sites préhistoriques, les sites à Ours des cavernes ont également été pris en compte, afin de replacer Regourdou dans le contexte général des théories sur les relations entre Hommes préhistoriques et Ours.

Bien qu'il s'en défende (Bonifay E. 2002 - p. 252, 2008 p. 29), l'interprétation de Regourdou donnée par son fouilleur ne peut que rappeler la théorie du «Culte de l'Ours » véhiculée dans la première moitié du $X X^{e}$ siècle par Emil Bächler (Bächler 1920-1921, 1923, 1929, 1940). A partir des fouilles qu'il a dirigées dans deux grottes alpines, Drachenloch (Bächler 1920-1921, 1923, 1929, 1940) et Wildmannlisloch (Bächler 1940), ce préhistorien suisse a défini une culture propre aux Alpes et à l'interglaciaire RissWürmien, le «Paléolithique alpin » ou « Moustérien alpin ». Selon lui, cette culture se caractériserait par : une chasse spécialisée aux Ours des cavernes juvéniles (oursons), l'été, dans des sites d'altitude ; la fabrication d'un outillage en os, obtenu par polissage, et d'une industrie lithique, plus primitifs que dans le Moustérien Quina ; et un "Culte de l'Ours des cavernes ", " culte de la chasse et du sacrifice " (Bächler 1920-1921 - p. 108-110), se manifestant par des dépôts d'os longs et de crânes d'ours des cavernes (position anthropique des ossements - contre les parois, enchevêtrement...- et répartition dans des structures -sépultures et caissons en pierres à la Drachenloch, murets ou cistes à la Wildmannlisloch). Cette théorie a connu rapidement un grand succès, permettant la propagation géographique de la culture du « Paléolithique alpin » en Allemagne, Autriche, Hongrie, Slovénie, Croatie..., grâce à de nombreuses découvertes similaires de dépôts intentionnels d'ossements d'ours (Pacher 2002 - p. 236240).

Après la remise en cause de l'origine anthropique du matériel par plusieurs chercheurs, notamment F.E. Koby (travaux en paléontologie, publiés entre 1942 et 1953, notamment sur le " charriage à sec ») et J.P. Jéquier (19621967 ; monographie consacrée à l'industrie lithique, publiée en 1975), la notion de "Culte de l'Ours » a été presque totalement abandonnée, hormis quelques réminiscences (Lajoux 1996, 2006 ; Lascu et al. 1994). Toutefois, de nombreux témoignages de relation ayant uni les Hommes aux Ours sont aujourd'hui connus, grâce à la découverte de stries de découpe sur des ossements
d'Ursidés provenant de sites récemment fouillés ou du réexamen de matériel issu de fouilles plus anciennes. Ces témoignages sont de plusieurs natures : si certaines actions très probablement anthropiques observées à Chauvet (déplacements d'ossements d'ours des cavernes attestés par une position non naturelle (os plantés dans le sol, dans une fissure pariétale...) ou par des négatifs sur le sol calcité, Philippe et al. 2001 - p. 49-56) ou à Taubach (destruction systématique de $80 \%$ des canines d'ours bruns, Kürten 1976 - p.105), ne sont pas explicables dans l'état actuel des connaissances autrement que par le terme vague de "symbolique ", la grande majorité des témoignages est de type utilitaire. La principale difficulté est alors d'évaluer la part de la chasse et du charognage dans cette exploitation.

Ainsi, les stries de découpe découvertes sur des ossements d'Ursus thibetanus de la grotte des Cèdres (Var) pourraient indiquer une activité de dépouillement ou une éventuelle utilisation alimentaire du pied (Bez 1995 - p. 4344), sans qu'il soit possible de déterminer si les hommes du Paléolithique moyen ont amené eux-mêmes la carcasse ou s'ils ne sont intervenus que secondairement. L'hypothèse d'un charognage dans un lieu de mort naturelle d'ours spéléens semble privilégiée pour les sites moustériens de Madonna dell'Arma et Badalucco (Italie) (Quiles 2003), Le Portel (Ariège) (Gardeisen 1994), Goyet (Belgique) (Germompré et Sablin 2001), Salzofen (Autriche) (Ehrenberg 1958-59, cité In Armand et al. 2004) et Divje Babe (Slovénie) (Turk 1997), mais aussi pour certains sites du Paléolithique supérieur, comme la grotte aurignacienne Pauline (Belgique) (Cordy 1974) ou le niveau gravettien du site allemand de Geissenklösterle (Münzel et al. 2001). Certains sites sont plus ambigus à interpréter, comme les deux niveaux châtelperronien (niveau $\mathrm{Xc}$ ) et aurignacien (VII) de la grotte du Renne d'Arcy-sur-Cure (Yonne) (David 2002), dans lesquels des ours morts naturellement coexistent avec des ours exploités par l'homme. Dans la galerie châtelperronienne Font-de-Gaume II (Dordogne), l'exploitation anthropique, hors contexte d'habitat, de 3 des 10 ours présents incite à penser que "les hommes [sont] venus spécialement pour exploiter les ours : que ceux-ci soient morts naturellement ou qu'ils aient été tués, l'exploitation relève d'une action planifiée » (Armand et al. 2004 $\mathrm{p}$.105). Cette hypothèse exclurait un charognage opportuniste. Sur le site italien de la Caverna delle Fate, des stries sur un os hyoïde pourraient témoigner de l'extraction de la langue par les Moustériens (Quiles 2003 p.548), opération nécessitant une certaine rapidité après la mort du plantigrade. Enfin, la chasse à l'Ours des cavernes n'est attestée, semble-t-il, que sur le site d'Höhle Fels (Allemagne) qui a livré, à l'intérieur d'un niveau gravettien, une vertèbre dorsale d'ours des cavernes portant un impact de silex dans le processus transverse (Münzel et al. 2001).

Des traces d'exploitation anthropique en contexte de mort naturelle ont également été découvertes sur une partie des rares restes d'ours brun mis au jour sur trois sites moustériens, Hayonim (Israël), Moscerini et Sant'Agostino (Italie), et un site épigravettien, Polesini (Italie) (Stiner 1994 p. 111 ; 2002 - p.158). Au contraire, deux sites mousté- 
riens, Biache-Saint-Vaast (Pas-de-Calais) (Auguste 1995a, 1995b) et Taubach (Allemagne), ont livré d'importantes accumulations d'ossements d'ours brun (2243 ossements à Biache, 1537 à Taubach), vraisemblablement d'origine anthropique. Cette interprétation se base sur plusieurs critères : prédominance des adultes au détriment des individus jeunes et âgés, structure contraire à celle d'une population vivante (adultes minoritaires, individus jeunes et âgés majoritaires) ; traces de découpe indiquant des activités de boucherie diversifiées (dépouillement (récupération de la peau ou de la fourrure), désarticulation et décharnement (récupération de la viande)). Sur le site de Taubach, l'extraction de la langue a également été observée ainsi qu'une recherche de moelle, les os se réduisant souvent à une extrémité ou à une diaphyse suite à une intense fracturation anthropique. S'il n'a pas été possible de déterminer avec certitude l'origine anthropique des Ursidés de ce site allemand, cette hypothèse apparaît comme la plus probante.

Ainsi, les ours de Taubach ont été exploités rapidement après leur mort (Bratlund 1999 - p. 118), tandis qu'à Biache les chercheurs penchent pour l'hypothèse d'une chasse opportuniste : les Moustériens auraient profité du terrain marécageux pour rendre vulnérables des animaux, dangereux en terrain sec (Ours, mais aussi Aurochs et Rhinocéros), venus se désaltérer au cours d'eau, à proximité du gisement (Auguste 1995b - p.163). Bien que l'hypothèse d'une chasse à l'ours soit la plus probable pour les auteurs, le seul témoignage de chasse préhistorique à l'ours brun reconnu unanimement à l'heure actuelle provient du site azilien du Bichon (Suisse). En effet, les deux squelettes partiellement entremêlés d'une femelle ours d'environ cinq ans et d'un jeune Homo sapiens âgé d'une vingtaine d'années (entre 20 et 23 ans) provenant de cette petite cavité ont été interprétés comme résultant d'un accident de chasse. Cette interprétation se base notamment sur la présence d'un impact de projectile lithique dans la troisième vertèbre cervicale de l'ourse, qui aurait occasionné une blessure fatale, comme le prouve l'absence de traces de régénération du tissu osseux. Le scénario admis est celui d'une ourse qui, mortellement blessée à l'extérieur de la cavité, réussit à se réfugier dans sa tanière. Après une possible tentative d'enfumage, que semblent indiquer les très nombreux charbons de bois retrouvés, le chasseur se serait alors engagé dans la grotte : arrivé à hauteur de l'animal, ce dernier lui aurait asséné un coup fatal avant de succomber à ses propres blessures (Chauvière et al. 2008).

Les comparaisons réalisées avec Regourdou n'ont pas été significatives pour les sites anthropiques, que ce soit en terme de représentation squelettique (Regourdou : surreprésentation des éléments dentaires et des carpo-tarsiens ; Biache-Saint-Vaast : légère sur-représentation des os des pattes ; Taubach : sur-représentation des carpo-tarsiens (plus de $50 \%$ ) et des restes dentaires (plus de $30 \%$ ), sous-représentation du squelette axial et de la partie supérieure des membres) ou de fréquence des stries de découpe (Regourdou : les trois ossements d'ours bruns portant des stries ne représentant que 0,08 \% du NRD et $0,07 \%$ du matériel total ; Biache-Saint-Vaast : stries pré- sentes sur $10 \%$ des restes d'ursidés, en majorité sur des éléments du squelette crânien et thoracique ; Taubach : stries visibles sur de très nombreux ossements -maxillaires et mandibules, vertèbres, os longs, pelvis, calcanéums, os du métapode, phalanges). Au contraire, cette très faible fréquence des témoignages anthropiques peut être comparée aux sites étudiés par M.C. Stiner, qui n'ont livré que très peu de restes d'ours portant des stries : une seule phalange à Moscerini et à Sant'Agostino (Italie), plusieurs à Hayonim (Israël), associées à des traces de combustion, et un fragment fémoral à Polesini (Italie) (Stiner 1994 - p.111 ; 2002 p.158). Néanmoins, contrairement au site de Regourdou sur lequel l'Ours brun représente près des deux tiers (62 \%) de l'assemblage faunique, ces sites n'ont livré que de rares restes d'ours brun.

\section{Conclusions générales}

Le site de Regourdou présente un grand intérêt archéologique, matérialisé par la présence d'une sépulture néandertalienne, peut-être la plus ancienne d'Europe, et par la prédominance de l'Ours brun (65\% NRD) sur le matériel faunique, taxon plutôt rare dans un site préhistorique. L'étude de ce site a souffert d'un contexte historique particulier, la publication des données ayant été freinée par l'interruption, pensée temporaire, des fouilles.

Cette étude a mis en évidence la grande difficulté de compréhension du site de Regourdou. L'interprétation de cette accumulation comme étant d'origine entièrement anthropique se basait pour une grande part sur le nombre très important de restes d'ours bruns. Cette prédominance est en effet plutôt inhabituelle dans un site pléistocène, d'origine anthropique ou naturelle, de plein air ou en grotte, l'Ursidé majoritairement représenté dans les spectres fauniques étant Ursus spelaeus. De cette représentation différentielle, probablement due à une différence comportementale (l'Ours des cavernes semblant avoir hiberné uniquement dans des grottes profondes tandis que l'Ours brun, dont l'éthologie au cours de la préhistoire est mal connue, peut occuper un abri moins protégé), a découlé I'hypothèse d'une origine assurément anthropique de toutes les accumulations d'ossements d'Ursus arctos. Toutefois, des observations actuelles attestent l'utilisation de grottes comme tanières par les ours bruns, notamment par les femelles en gestation ou suitées (les jeunes pouvant rester avec leur mère jusqu'à deux ans) (Camarra 1989) : il est donc possible que la grotte de Regourdou ait été principalement utilisée comme tanière, son occupation par les Moustériens n'étant attestée que pour la couche IV.

Malgré la présence de facteurs pouvant apparaitre contradictoires (tels que le nombre important de restes d'herbivores (NR : 1201), dont l'étude taphonomique semble indiquer une origine naturelle -hypothèse d'un piégeage naturel-), l'hypothèse d'une utilisation du site comme tanière, au moins dans la partie inférieure de la stratigraphie, semble probante, corroborée par plusieurs constatations (notamment taphonomiques) réalisées sur le matériel faunique de Regourdou. Néanmoins, l'intervention humaine est attestée ponctuellement par des stries de découpe pré- 
sentes sur seulement sept vestiges dispersés spatialement (couches II, IV, VII, VIIA et VIIIA) et par des os d'ongulés (Renne) présentant une fracturation d'origine, très probablement anthropique (couche II). A l'intérieur de la couche IV, dans laquelle se trouvait la sépulture néandertalienne, une exploitation, au moins utilitaire (récupération de la fourrure et/ou de la chair), de l'ours par l'Homme de Néandertal est attestée, grâce à la découverte d'un tibia et d'une côte portant plusieurs marques de découpe. Cette constatation rapproche Regourdou de plusieurs autres sites sur lesquels des découvertes similaires d'ossements d'ours bruns portant des stries anthropiques ont été réalisées, notamment Hayonim (Israël), Moscerini, Sant'Agostino et Polesini (Italie) (Stiner 1994), et, dans une moindre mesure, Biache-SaintVaast (Pas-de-Calais) (Auguste 1995a, 1995b) et Taubach (Allemagne) (Bratlund 1999 ; Kürten 1977).

Malgré le faible nombre d'ossements striés, la relation Homme/Ours semble être une composante majeure du site, les Moustériens ayant choisi, semble-t-il, d'inhumer l'un des leurs dans une grotte à ours. En effet, Regourdou est le seul site à montrer une telle association Homme préhistorique/Ours en contexte funéraire ; un autre site, Pestera cu Oase (Roumanie), a livré des restes humains dans un lieu d'hibernation d'ours des cavernes (Quiles et al. 2006) mais pas en contexte funéraire.

Cette deuxième étude du matériel faunique du site de Regourdou ne permettra donc pas de répondre, avec une absolue certitude, à la question de l'origine des ossements d'ours bruns retrouvés en masse. Son intérêt aura été de mettre en valeur plusieurs constatations laissant penser à une mortalité naturelle des ours bruns in situ et, éventuellement à une utilisation du site comme tanière. Néanmoins, de nombreux points doivent encore être approfondis. En premier lieu, l'origine du matériel des Herbivores, retrouvé récemment, devra être déterminée (naturelle, type avenpiège ou anthropique), afin de mieux comprendre le fonctionnement du site et son histoire taphonomique. La structure démographique de la population d'Ursidés devra être confirmée ultérieurement par l'établissement de classes d'âges plus précises, notamment pour les sub-adultes.

Tous ces éléments aideront à la détermination de la fonction (tanière ou aven-piège ?) de ce site, dont la complexité est encore renforcée par la découverte de nouveaux restes humains (Madelaine et al. 2008), complétant parfaitement les membres inférieurs du squelette néandertalien et dont un reste au moins (le fémur) semble porter des stigmates de charognage par l'ours. Cette observation semble corroborer l'hypothèse d'une porosité des structures de la couche IV, hypothèse émise face au nombre important de réajustements (appariements, raccords et remontages) réalisés à l'intérieur d'un même niveau, entre vestiges retrouvés dans et hors structures. Le charognage d'un vestige humain tendrait en effet à prouver l'intrusion, voire la circulation, des ours à l'intérieur de la sépulture (tumulus IVA). Dès lors, de nouvelles hypothèses peuvent être émises quant à l'absence remarquée du crâne néandertalien : il est effectivement possible d'imaginer que le calvarium puisse être toujours présent in situ, les ours ayant pu le déplacer hors de la zone sépulcrale elle-même.

\section{Remerciements}

Je tiens tout d'abord à exprimer ma gratitude envers Monsieur Eugène Bonifay, Directeur de recherche honoraire au CNRS, qui m'a aimablement autorisé à étudier ce site qui lui tient particulièrement à cœur, et envers Madame Marie-Françoise Bonifay, chargée de recherche honoraire au CNRS, pour avoir accepté si gentiment de confronter nos données. Je tiens également à remercier Monsieur Jean-Jacques Cleyet-Merle, conservateur en chef du MNP, et Madame Michèle Constant, propriétaire actuelle du site de Regourdou, sans qui l'étude du matériel n'aurait pu se faire. Enfin, je remercie Philippe Fosse pour m'avoir guidée tout au long de cette étude et Stéphane Madelaine pour son aide toujours chaleureuse durant les nombreux séjours d'étude effectués aux Eyzies. Je tiens également à remercier Mesdames Françoise Delpech et Marie-Françoise Bonifay pour les précieuses corrections qu'elles ont apportées à cet article.

\section{Références bibliographiques}

ALTUNA J. 1973 - Hallazgos de Oso Pardo (Ursus arctos, Mammalia) en cuevas del Pais Vasco. Munibe, XXV (2-3), p.121-170.

ARMAND D., PLASSARD F. et PRAT F. 2003 - L'ours des cavernes de Font-de-Gaume II. Paléo, 15, p. 241-244.

AUGUSTE P. 1995a - Cadres biostratigraphiques et paléoécologiques du peuplement humain dans la France septentrionale durant le Pléistocène. Apports de l'étude paléontologique des grands mammifères du gisement de Biache-Saint-Vaast (Pas-de-Calais). Thèse de $3^{\text {e }}$ cycle, Paris : Institut de Paléontologie Humaine, 1995. 1925 p.

AUGUSTE P. 1995b - Chasse et charognage au paléolithique moyen ; l'apport du gisement de Biache-Saint-Vaast (Pas-de-Calais). Bulletin de la Société Préhistorique Française, 92 (2), p. 155-167.

AVERY G., AVERY D.M., BRAINE S. et LOUTIT R. 1984 Bone accumulation by hyaenas and jackals : a taphonomic study. South African Journal of Science, 80, p. 186-187.

BÄCHLER E. 1920-1921 - Das Drachenloch ob Vättis im Taminatale, $2445 \mathrm{~m}$ ü.M. und seine Bedeutung als paläontologische Fundstätte und prähistoriche Niederlassung aus der Altsteinzeit (Paläolithikum) im Schweizerland. Jahrbuch der St Gallischen Naturwissenschaftlichen Gesellschaft, 59, p. 1-144.

BÄCHLER E. 1923 - Die Forschungsergebnisse im Drachenloch ob Vättis im Taminatale 2445 m ü.M Nachtrag und Zusammenfassung. Jahrbuch der St Gallischen Naturwissenschaftlichen Gesellschaft, 59, p. 79-118.

BÄCHLER E. 1929 - Die ältesten Knochenwerkzeuge insbesondere des alpinen Paläolithikums. Jahrbuch der Schweizerischen St Gallischen Gesellschaft für U- und Frühgeschichte, 20, 124-141. 
BÄCHLER E. 1940 - Das alpine Paläolithikum der Schweiz im Wildkirchli, Drachenloch und Wildenmanlisloch. Basel Monographien zur U- und Frühgeschichte der Schweiz, $263 \mathrm{p}$.

BEZ J.-F. 1995 - Traces de boucherie sur les restes de grands mammifères. In : Defleur $\mathrm{A}$. et Cregut-Bonnoure $\mathrm{E}$. (éd.), Le gisement Paléolithique moyen de la grotte des Cèdres (Var). D.A.F., p. 43-47.

BONIFAY E. 1963 - La grotte du Régourdou (Montignac, Dordogne). Stratigraphie et industrie lithique moustérienne. L'Anthropologie, 68 (1-2), p. 49-64.

BONIFAY E. 2002 - L'homme de Neandertal et l'ours (Ursus arctos) dans la grotte du Régourdou (Montignac-surVézère, Dordogne, France). In : Tillet T., Binford L.R. (éds), L'ours et l'homme. Colloque d'Auberives 1997, p. 247-254.

BONIFAY E. 2008 - Le site du Régourdou (Montignac-surVézère, Dordogne) et le problème de la signification des sépultures néandertaliennes. Bulletin de la Société d'études et de recherches préhistoriques des Eyzies, 58, p. 25-31.

BONIFAY E. et VANDERMEERSCH B. 1962 - Dépôt rituel d'ossements d'ours dans le gisement moustérien du Régourdou (Montignac, Dordogne). Compte rendu de l'Académie des Sciences de Paris, 255, p. 1635-1636.

BONIFAY E., VANDERMEERSCH B., COUTURE C. et PANATONNI R. 2007 - La sépulture néandertalienne du Regourdou (Montignac-sur-Vezère, Dordogne). Mercuès : Centre d'Etude et de Recherche sur les Lacs, Anciens lacs et Tourbières du Massif-Central, $18 \mathrm{p}$.

BONIFAY M.F. 1989 - Analyse taphonomique des Ursidés de la grotte sépulcrale néanderthalienne du Régourdou (Dordogne, France). In : Otte M. (Ed.), L'homme de Néanderthal. Colloque international de Liège (4-7 décembre 1986) : Eraul, 6, p. 45-47.

BONIFAY M.F. 2008 - Importance de la population d'Ours bruns du Régourdou. Bulletin de la Société d'études et de recherches préhistoriques des Eyzies, 57, p. 14-24.

BRATLUND B. 1999 - Taubach revisited. Jahrbuch des Römisch-germanischen Zentralmuseums Mainz, 46, p. 61-174.

CAMARRA J.-J. 1989 - L'ours brun. Paris : éds. Hatier, 325 p.

CASTEL J.C. 2004 - L'influence des canidés sur la formation des ensembles archéologiques. Caractérisation des destructions dues au loup. Revue de Paléobiologie, 23, p. 675-693.

CAVANHIÉ N. 2007 - Etude archéozoologique et taphonomique des grands Carnivores du site Paléolithique moyen de Regourdou (Montignac, Dordogne). Mémoire de Master 2 mention Archéologie, spécialité Arts et Cultures de la Préhistoire, Université Toulouse 2 Le Mirail, 109 p.

CHAGNEAU J. 1985 - Contribution à l'étude des os des extrémités des pattes d'Ursus deningeri romeviensis.
Comparaison avec Ursus arctos et Ursus spelaeus. Bulletin de la Société d'Anthropologie du Sud-Ouest, 20 (2-3), p. 61-107.

CHALINE J. 1972 - Les Rongeurs du pléistocène moyen et supérieur de France. Paris : éd. CNRS, Cahiers de Paléontologie, $410 \mathrm{p}$.

CHAUVIÈRE F.X. (dir.), EGLOFF M. et JACQUAT M. 2008 La grotte du Bichon : un site préhistorique des montagnes neuchâteloises. Neuchatel : Archéologie neuchâteloise $\mathrm{n}^{\circ} 42,164 \mathrm{p}$

CORDY J.M. 1974 - La faune aurignacienne de la grotte Princesse Pauline à Marche-les-Dames. Bulletin de la Société Royale Belge d'Anthropologie et de Préhistoire, 85, p. 243-252.

COUTURIER M. 1954 - L'ours brun : «Ursus arctos Linnaeus», l'auteur, Grenoble, 904.

DAVID F. 2002 - Les ours du Châtelperronien de la grotte du Renne à Arcy-sur-Cure (Yonne). In : Tillet T., Binford L.R. (éds), L'ours et l'homme. Colloque d'Auberives 1997, p. 185-191.

DELPECH F. 1996 - L'environnement animal des Moustériens Quina du Périgord. Paléo, 8, p. 31-46.

DITTRICH L. 1960 - Milchgebi entwicklung und Zahnwechsel beim Braunbären (Ursus arctos L.) und anderen Ursiden. Gegenbaurs Morphologisches Jahrbuch, 101 (1), p. 1-141.

DOMINGUEZ-RODRIGO M. et PIQUERAS A. 2003 - The use of tooth pits to identify Carnivore taxa in tooth marked archaeofaunas and their relevance to reconstruct hominid carcass processing behaviours. Journal of Archaeological Science, 30, p. 1385-1391.

DONARD E. 1982 - Recherches sur les Léporinés quaternaires (Pléistocène moyen et supérieur, Holocène). Thèse de Géologie du Quaternaire et Préhistoire, Université Bordeaux I, n 1764, 1982, $165 \mathrm{p}$.

DONAT-AYACHE B. 2003 - Croissance crânienne et éruption dentaire d'Ursus arctos (Mammalia, Carnivora). Application aux sites du Mont Ventoux (Brantes, Vaucluse). Aix Marseille : 2003. 3 vols : 332 p. Thèse de doctorat Université Aix Marseille II.

EHRENBERG K. 1958-1959 - Die paläontologische, prähistoriche und paläo-ethnologische Bedeutung der Salzofenhöhle im Lichte der letzten Forschungen. Quartär, 10/11, p. $19-58$.

FOSSE P., MOREL P. et BRUGAL J.P. 2002 - Taphonomie et éthologie des Ursidés Pléistocènes. In : Tillet T., Binford L.R. (éds), L'ours et l'homme. Colloque d'Auberives 1997, p. 79-101.

GARDEISEN A. 1994 - Restes fauniques et stratégies de chasse dans le Pléistocène supérieur de la grotte ouest du 
Portel (Ariège, France). Université Paul Valery (Montpellier III) : 1994.484 p. Thèse de Doctorat.

GERMOMPRÉ M. et SABLIN M.V. 2001 - The cave bear (Ursus spelaeus) from Goyet, Belgique. Bulletin de l'Institut royal des Sciences naturelles de Belgique Sciences de la terre, 71, p. 209-233.

GIFFORD-GONZALEZ D. 1989 - Ethnographic analogues for interpreting modified bones : some cases of East Africa. In : Bonnischen R., Sorg M.H. (éds) - Bone modification. Orono, University of Maine : Center for the Study of the First Americans, p. 179-246.

HARLE E. 1982 - Présentation d'os de repas d'hyènes tachetées. Bulletin de la société d'Histoire naturelle de Toulouse, 26, p. 22-25.

HAYNES G. 1980 - Evidence of Carnivore gnawing on Pleistocene and recent mammalian bones. Paleobiology, 6, p. 341-351.

HAYNES G. 1983 - A guide for differentiating mammalian Carnivore taxa responsible for gnaw damage to herbivore limb bones. Paléobiology, 9/2, p. 164-172.

HENSCHEL J.R. et TILSON R. 1988 - How much do a spotted hyaena eat? Perspective from the Namib desert. African Journal of Ecology, 26, p. 247-255.

HILL A. 1989 - Bone modification by modern spotted hyenas. In : Bonnischen R., Sorg M.H. (éds) - Bone modification. p. 169-178.

JÉQUIER J.P. 1975 - Le Moustérien alpin : révision critique. Neuchatel : Institut d'Archéologie Yverdonnoise, 106 p.

KLEIN R.G. 1975 - Paleanthropological implications of the nonarcheological bone assemblage from Swartklip I, South-Western Cape Province, South Africa. Quaternary Research, 5, p. 275-288.

KLEIN R.G., CRUZ-URIBE K. et BEAUMONT P.B. 1991 Environmental, ecological et paleoanthropological implications of the late Pleistocene mammalian fauna from Equus Cave, northern Cape Province, South Africa. Quaternary Research, 36, p. 94-119.

KOBY F.E. 1942 - Les soi-disants instruments osseux du paléolithique alpin et le charriage à sec des os d'ours des cavernes. Verhandlungen der Naturforschenden Gesellschaft in Basel, LIV, p. 59-97.

KOBY F.E. 1949 - Le dimorphisme sexuel des canines d'Ursus arctos et d'U. spelaeus. Revue Suisse de Zoologie, 56 , p. $675-687$.

KOBY F.E. 1951a - Grottes autrichiennes avec culte de l'ours ? Bulletin de la Société Préhistorique Française, 48, p. 8-9.

KOBY F.E. 1951b - L'ours des cavernes et les paléolithiques. L'Anthropologie, 55 (3-4), p. 304-308.
KOBY F.E. 1953 - Les paléolithiques ont-ils chassé l'ours des cavernes ? Actes de la Société Jurassienne d'Emulation, p. 1-48.

KÜRTEN B. 1976 - The Cave Bear story. Life and Death of a Vanished Animal. New-York : Columbia University Press, $163 \mathrm{p}$.

KÜRTEN B. 1977 - Bären- und Hyänenreste dem Pleistozän von Taubach. Quartärpaläontologie, 2, p. 361-378.

LAJOUX J.D. 1996 - L'homme et l'ours. Grenoble : Glénat, $224 \mathrm{p}$.

LAJOUX J.D. 2006 - Le culte de l'ours : un souvenir de la préhistoire ? Archéologia, 438, p. 62-70.

LASCU C., BACIU F., GLIGAN M. et SARBU S. 1994 - Cave bear worship site in Pestera Rece, Bihor Mountains, Romania. Theoretical and Applied Karstology, 7, p. 163-172.

LEROI-GOURHAN A. 1947 - La grotte des Furtins (Commune de Berzé-la-Ville, Saône-et-Loire). Bulletin de la Société Préhistorique Française, 44 (1-2), p. 43-55.

LOLLIOT S. et PHILIPPE M. 2004 - Note préliminaire sur les traces de charognage affectant les ossements d'ours des cavernes, Ursus spelaeus, de la Balme à Collomb (Entremont-le-Vieux, Savoie) ; en exemple : le cas des fémurs. Cahiers scientifiques, Hors-série $\mathrm{n}^{\circ} 2$ Actes du 9e Symposium international sur l'ours des Cavernes, p. 115-120.

MADELAINE S., MAUREILLE B., CAVANHIÉ N., COUTURE-VESCHAMBRE C., ARMAND D., BONIFAY M.F., DUDAY H., FOSSE Ph., VANDERMEERSCH B. et BONIFAY E. 2008 - Nouveaux restes humains moustériens rapportés au squelette néandertalien de Regourdou 1 (Regourdou, commune de Montignac, Dordogne, France). Paléo, 20, 2008, p. 101-114.

MAUREILLE B., ROUGIER H., HOUET F. et VANDERMEERSCH B. 2001 - Les dents inférieures du Néandertalien Regourdou 1 (site de Regourdou, commune de Montignac, Dordogne) : Analyses métriques et comparatives. Paléo, 13, 2001, p. 183-194.

MAUREILLE B. et VANDERMEERSCH B. 2007 - Les sépultures néandertaliennes. In : Vandermeersch $\mathrm{B}$. et Maureille B. (éds), 2007, Les Néandertaliens, biologie et Cultures. Documents préhistoriques 23, Paris : C.T.H.S, p. 311-322.

MÜNZEL S., LANGGUTH K., CONARD N. et UERPMANN H.P. 2001 - Höhlenbärenjagd auf der Schwäbischen Alb vor 30.000 Jahren (Cave Bears Hunting on the Schwäbischen Alb 30.000 Years Ago). Archäologisches Korrespondenzblatt, 31 (3), p. 317-328.

PACHER M. 2002 - Polémique autour d'un culte de l'ours des cavernes. In : Tillet T., Binford L.R. (éds), L'ours et l'homme. Colloque d'Auberives 1997, p. 235-246. 
PHILIPPE M., FOSSE P., GARCIA M. et GENESTE J.-M. 2001 - Les sols et la fréquentation de la caverne par les animaux et par les hommes. In : Clottes J. (éds) - La grotte Chauvet, l'art des origines. Paris : Seuil, p. 32-59.

PINTO LLONA A.C. et ANDREWS P.J. 2004 - Taphonomy and palaeoecology of Ursus spelaeus from northern Spain. Cahiers scientifiques, 9th International Cave Bear Symposium, Hors série $\mathrm{n}^{\circ} 2$, p. 163-170.

POPLIN F. 1980 - Le dépeçage des animaux. Les dossiers de l'archéologie : revivre la préhistoire, 46 (septoct), p. 24-32.

PRAT F. et THIBAULT C. 1976 - Le gisement de Nauterie à la Romieu (Gers) : fouilles de 1967 à 1973. Nauterie I. Mémoires du Muséum National d'Histoire Naturelle, vol. XXXV, $82 \mathrm{p}$.

QUILES J. 2003 - Les Ursidae du Pléistocène moyen et supérieur en Midi méditerranéen : Apports paléontologiques, biochronologiques et archéozoologiques. Paris : 2003. $610 \mathrm{p}$. Thèse de doctorat Muséum d'Histoire naturelle de Paris.

QUILES J., PETREA C., MOLDOVAN O., ZILHÃO J., RODRIGO R., ROUGIER H., CONSTANTIN S., MILOTA S., GHERASE M., SARCINÀ L. et TRINKAUS E. 2006 - Cave bears (Ursus spelaeus) from the Pestera cu Oase (Banat, Romania) : Paleobiology and taphonomy. Palevol, 5, p. 927-934.

SIMARD-PELISSIER S. 1966 - Etude paléontologique des micromammifères de la grotte du Régourdou. Faculté des Sciences de l'Université de Paris, 1966. 78 p. thèse de $3^{\text {e }}$ cycle, Géologie et Paléontologie.

SKINNER J.D., HENSCHEL J.R. et VAN JAARSVELD A.S. 1986 - Bone collecting by brown hyenas Hyaena brunnea in the central Namib desert. Journal of Archaeological Science, 18, p. 513-523.

STINER M.C. 1990 - The use of mortality patterns in archaeological studies of hominid predatory adaptations. Journal of Anthropological Archaeology, 9, p. 305-351.
STINER M.C. 1994 - Honor among thieves. A Zooarchaeological Study of Neandertal Ecology. Princeton : Princeton University Press, $447 \mathrm{p}$.

STINER M.C. 1998 - Reconstructing cave bear paleoecology from skeletons : a cross-siciplinary study of middle Pleistocene bears from Yarimburgaz Cave, Turkey. Paléobiology, 24 (1), p. 74-98.

STINER M.C. 2002 - Pourquoi ossements d'ours et outillages coexistent-ils dans les sites en grotte paléolithiques? Observations provenant du pourtour méditerranéen. In : Tillet T., Binford L.R. (éds), L'ours et l'homme. Colloque d'Auberives 1997, p. 157-165.

TILLIER A.M., ARENSBURG B., VANDERMEERSCH B. et RAK Y. 1991 - L'apport de Kébara à la paléthnologie funéraire des Néanderthaliens du Proche-Orient. In : Bar Yosef O., Vandermeersch B. (éds) - Le squeltte moustérien de Kébara 2. Paris : Cahiers de Paléoanthropologie, éditions du CNRS, p. 89-95.

TORRES PÉREZ HIDALGO (de) T. 1988 - « Osos (Mammalia, Carnivora, Ursidae) del Pleistoceno Iberico (Ursus deningeri Von Reichenau, U. spelaeus RosenmüllerHeinroth, U. arctos Linné) : V. Denticion decidual, formula dentaria y denticion superior", in Boletin de Géologia y Mineria, 99 (5) : 16-70.

TURK I. 1997 - Taxonomic and taphonomic survey of mammal macrofauna. In : Turk I., Bastiani G. (éds) Mousterian "Bone flute " and other finds from Divje Babe I cave site in Sloveni. p. 99-113.

TURQ A., JAUBERT J., MAUREILLE B., LAVILLE D. 2008 Le cas des sépultures néandertaliennes du Sud-Ouest : et si on les vieillissait ? In : Vandermeersch B., Cleyet-Merle J.-J., Jaubert J., Maureille B. et Turq A. (éds), Première humanité, gestes funéraires des Néandertaliens. Paris : Réunion des Musées Nationaux, p. 40-41.

VANDERMEERSCH B. et TRINKAUS E. 1995 - The postcranial remains of the Regourdou 1 Neandertal : the shoulder and arm remains. Journal of Human evolution, 28, p. $439-476$. 
